\title{
Nanoindentation derived elastic constants of carbon fibres and their nanostructural based predictions
}

\author{
Tamás Csanádia, ${ }^{a,}$, Dušan Németh ${ }^{\mathrm{a}}$, Chengyu Zhang ${ }^{\mathrm{b}}$, Ján Dusza ${ }^{\mathrm{a}, \mathrm{c}}$
}

\begin{abstract}
Elastic constants of single carbon fibres were estimated by a novel nanoindentation based method and subsequently predicted by modified two- and three-phase Eshelby-Mori-Tanaka (EMT) micromechanical models which takes into account both crystalline, amorphous phases and microvoids of the fibre structure. This case study was carried out on a T-300 PAN-based carbon fibre reinforced $\mathrm{SiC}$ composite material at room temperature. Transversal and longitudinal cross-sections of individual fibres were indented by a sharp Berkovich tip up to the maximum depth of $150 \mathrm{~nm}$. Assuming transverse isotropy of the fibres, their five elastic constants were deduced from the measured indentation moduli using numerical (Vlassak-Nix) and analytical
\end{abstract}


models (Delafrague-Ulm), and finite element method simulations. The elastic constants were estimated to $\mathrm{c}_{11}=30.9, \mathrm{c}_{12}=9.7, \mathrm{c}_{13}=12.2, \mathrm{c}_{33}=237.3$ and $\mathrm{c}_{44}=10.9 \mathrm{GPa}$. The complete set of measured elastic constants were analyzed and then predicted by the proposed modified EMT models. The application of anisotropic nanoindentation coupled with the amorphous-crystalline structure model is unique to carbon fibre which revealed that an anisotropic 'amorphous' matrix should be assumed to achieve an appropriate fit with experimental results. This matrix was considered as a mixture of microvoids and aligned graphite planes, and their volume ratio was determined.

\section{Introduction}

Carbon fibres play an important role in reinforcement of lightweight structures due to the combination of high strength and high modulus with a low weight. These kind of structural applications forming metal matrix composites are found in the aerospace industry, power plants (turbine blades) and increasingly in the automotive industry. In recent times, carbon fibres are also used as reinforcement in ceramic matrix composites, which are promising materials for advanced ceramics applications. Of particular interest are the carbon fibre woven silicon carbide composites $(\mathrm{C} / \mathrm{SiC})$ which possess high hardness and stiffness with enhanced toughness [1]. From the viewpoint of strengthening, the elastic properties of individual carbon fibres are determinative and their knowledge is important to predict and improve the macromechanical behaviour of these composites within their elastic limit $[2,3]$. The elastic properties of carbon fibres are strongly influenced by their nanostructure (e.g. shape, size and orientation distribution of graphite crystallites, amorphous carbon content, etc.) due to the different processing routes by which they are made $[4,5]$. They are considered transversely isotropic solids and characterized 
by five independent elastic constants which are in good agreement with ultrasound measurements. Carbon fibres are derived from several precursors and nowadays the polyacrylonitrile (PAN) based fibres are predominant.

In development of C-fibres, there are two distinct approaches to determine the elastic constants. On the one hand, the measurement of as prepared or embedded fibres inside the composite product, on the other hand, the prediction of fibre properties based on their measured nanostructure. The most common technique used for the measurement of a complete set of Cfibre elastic constants is the ultrasound. Most of these studies focused on the measurement of composite systems and the fibre properties were deduced from that by different methods [2, 6-9]. Another technique is the mechanical testing, which makes it possible to measure a few elastic constants of C-fibres, such as Young's modulus, transversal elastic modulus or shear modulus. These works include tensile tests combined with optical microscopy [10], laser diffraction [11, 12], X-ray diffraction [12-14], micro-Raman spectroscopy [15] on single carbon fibres or bundles, and nanoindentation studies [15-19] on C-fibre reinforced composites. In order to predict the C-fibre elastic properties, several models have been developed and improved during the last couple of decades $[10,12,14,20,21]$. Lately, the most accurate studies use micromechanical models taking into account both crystalline, amorphous and void content of $\mathrm{C}$ fibres [14, 21] and molecular dynamics simulations [20].

Despite the above mentioned approaches, the determination of elastic constants of carbon fibres is still challenging for several recently developed materials. Considering C-fibre woven ceramic matrix composites, such as $\mathrm{C} / \mathrm{SiC}$, the deduction of fibre elastic properties from an ultrasound measurement on composite is rather difficult. Conventional models work only for unidirectional fibre reinforced systems [6-8]. In the case of more complicated structures (e.g. woven or layered composites), the elastic constants of C-fibres are derived from different 
micromechanical models and finite element (FEM) simulations using an iterative method to get the best fit to the experimental stiffness tensor of the tested composite [2]. Measuring these properties on unidirectional fibre bundles prior to the composite prediction is also not always suitable due to their structural changes during high temperature sintering $[13,22]$. Instead, direct characterization of the embedded fibres is required, which can be carried out by micromechanical testing such as nanoindentation. The drawback of this method is the lack of a theoretical model to deduce the fibre elastic constants. Even the simulation approach is also not sufficient. Recent micromechanical models $[14,21]$ were developed to predict the Young's modulus of fibres and were not tested on the complete set of elastic constants.

The present work intends to further develop the mentioned testing and modelling approaches to determine the elastic constants of carbon fibres. The aim of our paper is to present an approximation of elastic constants for carbon fibres using a nanoindentation based method and to predict them by a proposed modified micromechanical model on the basis of fibre nanostructure. The present case study was carried out on a carbon fibre reinforced silicon carbide composite system (2D-C/SiC).

\section{Theoretical background}

\subsection{Approximation of $C$-fibre elastic constants by nanoindentation}

Nanoindentation is an efficient tool to determine local mechanical properties of materials including hardness, fracture and elastic properties as well. According to the standards, the effective elastic modulus $\left(E_{\text {eff }}\right)$ is evaluated from the measured load, displacement and stiffness $(S)$ data (see Eq. (1)) and simply related to the Young's modulus $(E)$ and Poisson's ratio (v) of the tested location assuming isotropic elasticity (Eq. (2)) [23]. 


$$
\begin{aligned}
& S=\beta \cdot \frac{2}{\sqrt{\pi}} \cdot \sqrt{A} \cdot E_{\text {eff }} \\
& \frac{1}{E_{\text {eff }}}=\frac{1-v_{i}^{2}}{E_{i}}+\frac{1-v^{2}}{E}
\end{aligned}
$$

In Eqs. (1) and (2), the meaning of $A$ is the projected area of elastic contact, $E_{i}$ and $v_{i}$ are the Young's modulus and Poisson's ratio of the indenter material, and $\beta$ is practically unity. The reciprocal of the second term on the right side in Eq. (2) is called an indentation modulus $(M)$. In an isotropic case, its form is $M=\frac{E}{1-v^{2}}$. The Young's modulus is calculated from the indentation modulus using Poisson's ratio of the material. The proper selection of $v$ value is not critical as it has only a slight influence on the evaluation [23]. Assuming anisotropic elasticity, the relation between the measured indentation modulus and the elastic constants $\left(c_{i j}\right)$ of the material is much more complicated. A theoretical model was proposed for the relationship between $c_{i j}$ and $M$ by Vlassak and Nix for flat punch and paraboloid tip geometries and later it was generalized for axisymmetric indenters with arbitrary shape [24, 25]. It was pointed out that the indentation modulus definition reported in [24] is applicable for a large class of anisotropic materials using axisymmetric indenters [25]. This $M$ formula is indicated in the Appendix A and only numerically can be evaluated using the $c_{i j}$ values of the material. In order to deduce the elastic constants from nanoindentation, an explicit expression is needed instead of that. In the work of Delefargue and Ulm [26], an approximate analytical expression of the indentation modulus of orthotropic materials was derived for conical indenters. It was obtained for the three directions perpendicular to the three plains of symmetry of orthotropic solids. As carbon fibres are assumed to be transversely isotropic, a subset of orthotropic materials with higher symmetry, the expressions reported by Delafargue [26] are applicable for them. In the case of carbon fibres, only two of those indentation modulus expressions are independent: the direction parallel with 
fibre axis, longitudinal direction $\left(M_{L}\right)$, and another one which is perpendicular to that, transversal direction $\left(M_{T}\right)$. These analytical expressions of indentation moduli for conical indenters, expressed by the stiffness coefficients of fibres, are as follows.

$$
\begin{gathered}
M_{L}=2 \cdot \sqrt{\frac{c_{11} \cdot c_{33}-c_{13}^{2}}{c_{11} \cdot\left(\frac{1}{c_{44}}+\frac{2}{\sqrt{c_{11} \cdot c_{33}}+c_{13}}\right)}} \\
M_{T}=2 \cdot \sqrt{\frac{c_{11}^{2}-c_{12}^{2}}{c_{33} \cdot\left(\frac{2}{c_{11}-c_{12}}+\frac{2}{c_{11}+c_{12}}\right)} \cdot \sqrt{\frac{c_{11} \cdot c_{33}-c_{13}^{2}}{c_{11} \cdot\left(\frac{1}{c_{44}}+\frac{2}{\sqrt{c_{11} \cdot c_{33}}+c_{13}}\right)}}}
\end{gathered}
$$

Here, the reference Cartesian coordinate system $\left(x_{1}, x_{2}, x_{3}\right)$ is selected so that the $x_{3}$ direction be parallel with the fibre axis. In this system, the five independent stiffness coefficients of carbon fibres are denoted by $c_{11}, c_{12}, c_{13}, c_{33}$ and $c_{44}$.

To determine all five elastic constants, an additional three experimental relationships are needed. One expression may be written for the Young's modulus of fibres in longitudinal direction $\left(E_{L}\right)$, along the fibre axis, which can be easily measured and is known by the supplier.

$$
E_{L}=c_{33}-\frac{2 \cdot c_{13}^{2}}{c_{11}+c_{12}}
$$

The other two relationships are the expressions of Poisson's ratios $\left(v_{T T}-\right.$ in transverse-transverse direction, $v_{L T}-$ in longitudinal-transverse direction), Eqs. (6), (7). These values have only a slight influence on indentation modulus, similarly to the isotropic case, and are assumed to be equal to $v_{T T}=v_{L T}=0.3$ based on experimental results $[6-8,11]$

$$
v_{T T}=\frac{c_{33} \cdot c_{12}-c_{13}^{2}}{c_{33} \cdot c_{11}-c_{13}^{2}}
$$




$$
v_{L T}=\frac{c_{13}}{c_{11}+c_{12}}
$$

It is important to note that practically only two independent elastic constants are measured with the present indentation method, the other three are derived from other sources. Therefore, nanoindentation cannot be considered as an alternative of the ultrasound technique itself. However, as it was described in the method, using additional measurements (e.g. tensile test, Xray, etc.), this may be completed to deduce the stiffness components of C-fibres.

\subsection{Nanostructure based micromechanical models}

In order to predict the elastic constants of carbon fibres, the Eshelby-Mori-Tanaka (EMT) micromechanical model was applied, which is one of the best analytical approximations of numerical FEM simulations [27]. This model was basically developed to calculate the elastic deformation of unidirectional fibre reinforced composites based on the properties of their micro constituents. Later on, it was generalized to take into account short fibres, more strengthening phases with different orientation distributions [27, 28]. As carbon fibres have a similar complex composite structure at nano level $[4,5]$, it was reasonable to adopt the EMT model for the description of their elastic behaviour [14, 21].

In this work, several assumptions were made similar to recent micromechanical models reported earlier [14, 21]. Firstly, the composition of carbon fibre is assumed to consist of three phases, crystalline carbon, amorphous matrix and microvoids. The schematic of the model is indicated in Fig. 1. Calculations were carried out for 2-phase (crystalline and amorphous) and 3phase (crystalline, amorphous and voids) models where the total volume fraction of each phase is equal to unity. Secondly, we assumed that the amorphous carbon matrix is isotropic which is 
characterized by its Young's modulus $\left(E_{m}\right)$ and Poisson's ratio $\left(v_{m}\right)$. The Poisson's ratio of isotropic matrix is fixed to $v_{m}=0.3$ and has a slight influence on the calculation. This assumption was changed later on, as is described in Section 5, which resulted in the proposed modified EMT model. Thirdly, the elastic constants of crystalline carbon is selected the same as those of graphite, which are $c_{11}=1060 \mathrm{GPa}, c_{12}=180 \mathrm{GPa}, c_{13}=15 \mathrm{GPa}, c_{33}=36.5 \mathrm{GPa}$ and $c_{44}=4 \mathrm{GPa}$ (index 3 coincides with the direction of the symmetry axis of the crystal) [29]. Fourthly, the shape of the crystallites is an oblate spheroid with the aspect ratio being equal to the ratio of crystallite thickness $L_{c}$ to diameter $L_{a}$, as $t_{c}=L_{c} / L_{a}$. The shape of the voids is a rotation ellipsoid with an aspect ratio of $t_{v}=L / a$ where $L$ is the half length of rotation axis and $a$ is he radius. These parameters were measured for several fibres by X-ray diffraction (XRD) and small angle X-ray scattering (SAXS) techniques [14, 21].

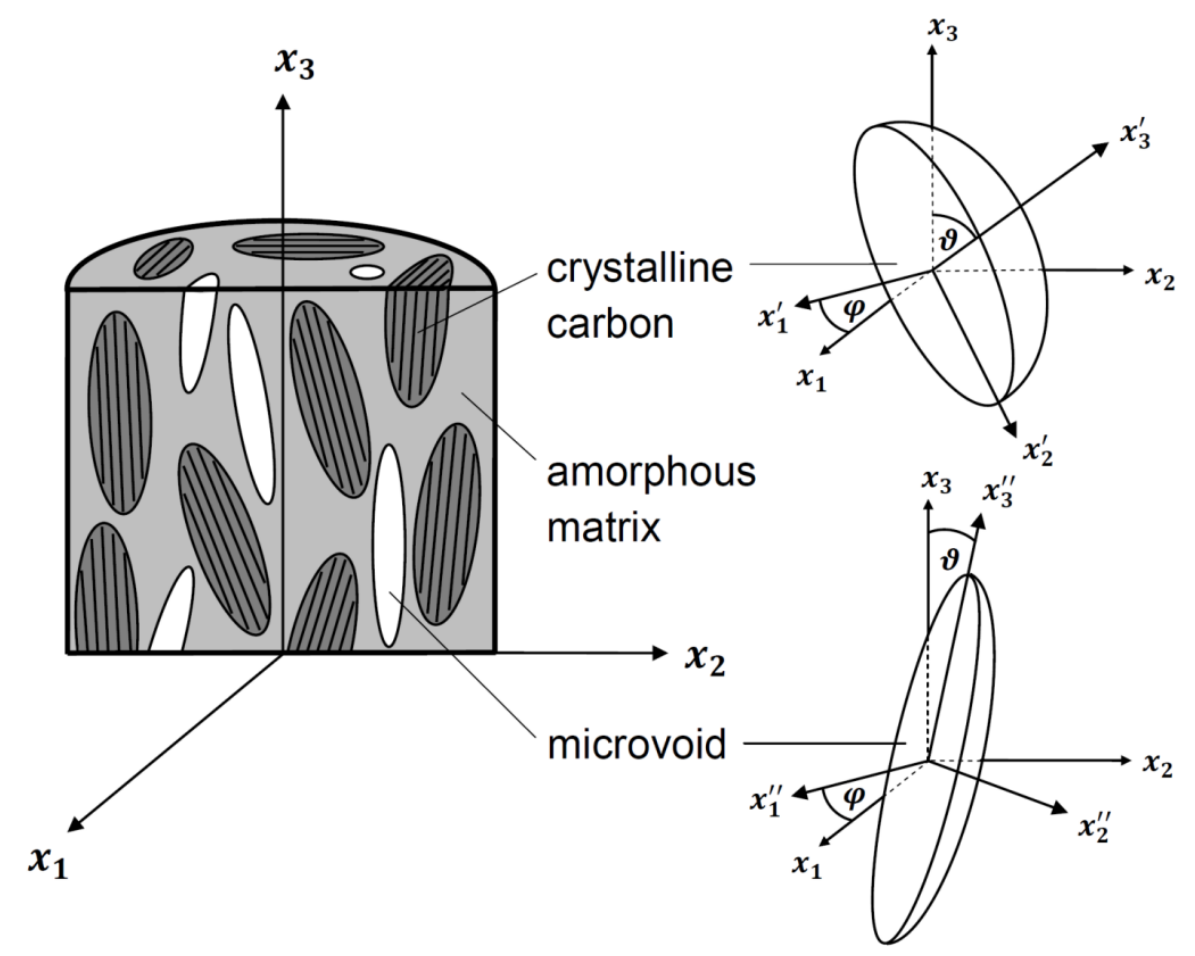

Fig. 1: The model of C-fibre structure consisting of amorphous matrix, crystalline carbon and microvoids together with the shape and the coordinate systems of the embedded phases. 
For a clear description of the model, three Cartesian coordinate systems are established as is shown in Fig. 1. The reference system $\left(x_{1}, x_{2}, x_{3}\right)$ is fixed to carbon fibre, such that the $x_{3}$ coincides with fibre axis (in agreement with the definition in Section 2.1). One of the local coordinate systems is defined for the crystalline carbon $\left(x_{1}{ }^{\prime}, x_{2}{ }^{\prime}, x_{3}{ }^{\prime}\right)$ where $x_{3}$ ' is selected to be parallel with the symmetry axis of the crystal. The other local system corresponds to the microvoid $\left(x_{1} ", x_{2} ", x_{3}\right.$ ”) where $x_{3}$ " coincides with the rotation axis of that. The stiffness tensor of amorphous matrix is independent of the coordinate system due to isotropy and is denoted by $C_{m}$. The stiffness tensor of crystalline carbon is signed by $C_{c 0}$ and defined in the coordinate system of the crystal $\left(x_{1}{ }^{\prime}, x_{2}{ }^{\prime}, x_{3}{ }^{\prime}\right)$. The components of the stiffness tensor of voids (indicated by $\left.C_{v 0}\right)$ are all zero, thus it is also independent of the coordinate system. The form of $C_{m}, C_{c 0}$ and $C_{v 0}$ are defined in Appendix B using the contracted Voigt notation. Based on the above assumptions, the expression of the averaged stiffness tensor using the EMT model for the more general 3-phase case is as follows.

$$
\bar{C}_{E M T}=\left(f_{m} \cdot C_{m}+f_{c} \cdot\left\{C_{c} \cdot A_{c}\right\}+f_{v} \cdot\left\{C_{v} \cdot A_{v}\right\}\right) \cdot\left(f_{0} \cdot I+f_{c} \cdot\left\{A_{c}\right\}+f_{v} \cdot\left\{A_{v}\right\}\right)^{-1}
$$

Here $f_{m}, f_{c}$ and $f_{v}$ represent the volume fraction of amorphous matrix, crystals and voids, respectively. The elastic constant tensors for each constituent are indicated by $C_{m}, C_{c}$ and $C_{v}$, the $I$ is the unit tensor. The calculation of averaged stiffness tensor was carried out in the reference Cartesian coordinate system $\left(x_{1}, x_{2}, x_{3}\right)$ on matrices of $6 \times 6$ using the Voigt contracted notation in the entire paper. As the crystals and voids have specific orientation distributions, those were averaged as is indicated by the below definition of curly brackets in Eq (8). The index $i$ refers to both crystalline and void phases. 


$$
\begin{gathered}
\left\{C_{i} \cdot A_{i}\right\}=\left\{T \cdot\left(C_{i 0} \cdot A_{i 0}\right) \cdot T^{T}\right\}=\frac{\iint_{0,0}^{2 \pi, \pi} T \cdot\left(C_{i 0} \cdot A_{i 0}\right) \cdot T^{T} \cdot \eta_{i}(\vartheta) \cdot \sin \vartheta d \vartheta d \varphi}{2 \cdot \pi \cdot \int_{0}^{\pi} \eta_{i}(\vartheta) \cdot \sin \vartheta d \vartheta} \\
\left\{A_{i}\right\}=\left\{\left(T^{-1}\right)^{T} \cdot A_{i 0} \cdot T^{T}\right\}=\frac{\iint_{0,0}^{2 \pi, \pi}\left(T^{-1}\right)^{T} \cdot A_{i 0} \cdot T^{T} \cdot \eta_{i}(\vartheta) \cdot \sin \vartheta d \vartheta d \varphi}{2 \cdot \pi \cdot \int_{0}^{\pi} \eta_{i}(\vartheta) \cdot \sin \vartheta d \vartheta}
\end{gathered}
$$

The $C_{i 0} \cdot A_{i 0}$ and $A_{i 0}$ quantities are defined in the coordinate system of crystals $\left(x_{1}{ }^{\prime}, x_{2}{ }^{\prime}, x_{3}{ }^{\prime}\right)$ and voids $\left(x_{1}{ }^{\prime}, x_{2}{ }^{\prime}, x_{3}{ }^{\prime}\right)$, respectively. In these expressions, $A_{c 0}$ and $A_{v 0}$ denote the so-called dilute stress concentration tensors for the crystals and microvoids which contain the Eshelby tensors $\left(E_{c}, E_{v}\right)$ and the compliance of the matrix $\left(S_{m}=C_{m}^{-1}\right)$. The Eshelby tensor depends on the size and the shape of the inclusions and is indicated in Appendix B [30].

$$
\begin{aligned}
& A_{c 0}=\left(I+E_{c} \cdot S_{m} \cdot\left(C_{c 0}-C_{m}\right)\right)^{-1} \\
& A_{v 0}=\left(I+E_{v} \cdot S_{m} \cdot\left(C_{v 0}-C_{m}\right)\right)^{-1}
\end{aligned}
$$

To obtain the averaged $\left\{C_{i} \cdot A_{i}\right\}$ and $\left\{A_{i}\right\}$ values in the reference system $\left(x_{1}, x_{2}, x_{3}\right)$, the $C_{i 0} \cdot A_{i 0}$ and $A_{i 0}$ tensors were subjected to a rotation by $T$ and then averaged according to their orientation distribution functions $\left(\eta_{i}(\vartheta)\right)$, shown in Eqs. (9), (10). The forms of $T, T^{T},\left(T^{-1}\right)^{T}$ and $\eta_{i}(\vartheta)$ are indicated in Appendix C.

\section{Experimental}

\subsection{Material}

The present case study was carried out on a carbon fibre reinforced silicon carbide composite system (2D-C/SiC). The $2 \mathrm{D}-\mathrm{C} / \mathrm{SiC}$ is reinforced by stacking plain weave carbon fibre cloths shown in Fig 2. The reinforcement is composed of 1000 polyacrylonitrile (PAN) based 
carbon filaments (Type: T-300, made in Toray Co, Japan) with a fibre diameter of $7 \mu \mathrm{m}$. A lowpressure chemical vapor infiltration (CVI) process was used to prepare the composites. The pyrolytic carbon $(\mathrm{PyC})$ interphase with a thickness of $0.2 \mu \mathrm{m}$ was deposited on carbon fibres with $\mathrm{C}_{3} \mathrm{H}_{6}$ at $1173 \mathrm{~K}$ and $5 \mathrm{kPa}$. Methyltrichlorosilane (MTS, $\mathrm{CH}_{3} \mathrm{SiCl}_{3}$ ) was used for depositing the $\mathrm{SiC}$ matrix. The applied temperature, the total pressure, and the molar ratio of $\mathrm{H}_{2}$ to MTS were $1000{ }^{\circ} \mathrm{C}, 5 \mathrm{kPa}$, and 10 , respectively. To achieve appropriate surface for testing, the sample was cut perpendicular to the plain weaves and subjected the conventional sample preparation (cutting, grinding, polishing) methods (see Fig. 2b).
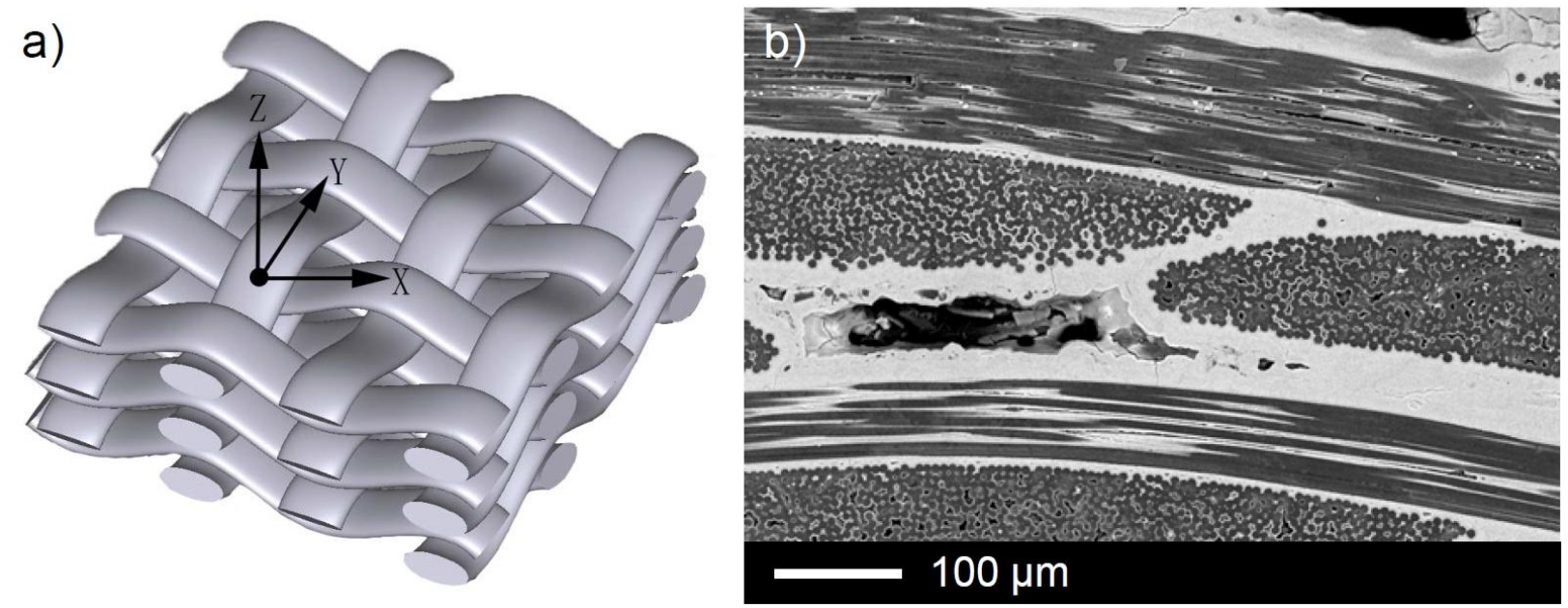

Fig. 2: a) Schematic of stacking plain weave carbon fibres embedded in silicon carbide matrix. b) Microstructure of a cross-section of the $2 \mathrm{D}-\mathrm{C} / \mathrm{SiC}$ composite material perpendicular to the carbon tows (in the direction of $X$ or $Y$ in Fig. 2a).

\subsection{Nanoindentation and surface analysis}

To determine the elastic constants of individual carbon fibres, nanoindentation was carried out on transversal and longitudinal cross-sections of fibres where the indentation direction is 
assumed to be parallel and perpendicular to the fibre axis, respectively. Selected surface areas covering these orientations were tested by an Agilent G200 NanoIndenter using a diamond Berkovich tip. Continuous stiffness measurement (CSM) mode was applied to register the depth, load and stiffness simultaneously during testing. On each orientation, 100 indents were prepared with a maximum depth of $150 \mathrm{~nm}$, strain rate of $0.05 \mathrm{~s}^{-1}$ and displacement between the indents of $1.8 \mu \mathrm{m}$. The hardness and modulus were automatically calculated according to the standards of instrumented indentation (Oliver-Pharr method [23]). The tip was calibrated prior to the measurement on fused silica which resulted in an accurate tip area function over $20 \mathrm{~nm}$. Poisson ratio and Young's modulus of the diamond tip were $v_{\mathrm{i}}=0.07$ and $E_{\mathrm{i}}=1140 \mathrm{GPa}$, respectively.

The surface topography of the tested areas and the individual indents were analyzed by scanning electron microscopy (SEM, Zeiss - Auriga) and atomic force microscopy (AFM, Veeco - Dimension Icon). These images were used to determine the inclination of fibres with respect to their exact transversal direction.

\subsection{Finite element simulation}

Finite element method (FEM) simulations were performed to determine the effect of indenter geometry and the influence of $\mathrm{SiC}$ matrix around the fibres upon the evaluation of indentation modulus. Simulations were performed by a 3D anisotropic elastic model using ANSYS software. Nanoindentation was simulated on transversal and longitudinal cross-sections of carbon fibres, embedded in a $\mathrm{SiC}$ matrix, with a fibre diameter of $7 \mu \mathrm{m}$ and maximum penetration depth of $150 \mathrm{~nm}$ in agreement with the experiments. Cubic sample geometry was used with side edges of $10 \mu \mathrm{m}$. Boundary conditions and mesh structure, which may have an influence on the calculation of indentation modulus, were applied based on our previous work 
[31]. In the 3D simulations, ideal conical and Berkovich tip geometries were used to approximate the real indentation conditions. The elastic constants of model filament were selected similar to T-300 fibre according to the work of Datta et al. [7] measured by ultrasound. For transversal cross-section, the elastic constants of fibre were defined as indicated in Tab. 1, then those were transformed to the longitudinal cross-section applying a $\vartheta=90^{\circ}$ rotation by $T_{x 1}$ tensor indicated in Appendix C. The elastic properties of isotropic $\mathrm{SiC}$ phase were selected to $E_{\mathrm{SiC}}=400 \mathrm{GPa}, v_{\mathrm{SiC}}=$ 0.18 while the tips were assumed to be rigid (i.e. $E_{t i p}=$ infinite) .

\begin{tabular}{|c|c|c|c|c|c|}
\hline \multirow{2}{*}{ Method } & \multicolumn{5}{|c|}{ Elastic constants, $c_{i j}[\mathrm{GPa}]$} \\
\hline & $c_{11}$ & $c_{12}$ & $c_{13}$ & $c_{33}$ & $c_{44}$ \\
\hline $\begin{array}{l}\text { ultrasound } \\
\text { ref. [7] }\end{array}$ & 20.99 & 7.98 & 9.77 & 225.17 & 17.99 \\
\hline $\begin{array}{l}\text { nanoindentation } \\
\text { this work }\end{array}$ & 30.9 & 9.7 & 12.2 & 237.3 & 10.9 \\
\hline $\begin{array}{l}\text { 2-phase EMT model } \\
\quad\left(\text { reduced } f_{c}\right)\end{array}$ & 151.0 & 50.0 & 54.0 & 260.5 & 74.4 \\
\hline $\begin{array}{l}\text { 2-phase EMT model } \\
\left.\text { (reduced } E_{m}\right)\end{array}$ & 91.0 & 30.6 & 28.9 & 244.9 & 62.6 \\
\hline $\begin{array}{l}\text { 3-phase EMT model } \\
\left(\text { reduced } f_{c}\right)\end{array}$ & 116.8 & 33.4 & 37.0 & 249.4 & 71.1 \\
\hline $\begin{array}{l}\text { 3-phase EMT model } \\
\left(\text { reduced } E_{m}\right)\end{array}$ & 79.8 & 23.2 & 21.9 & 240.4 & 63.0 \\
\hline $\begin{array}{l}\text { 2-phase modified } \\
\text { EMT model }\end{array}$ & 43.0 & 13.1 & 25.3 & 253.7 & 31.9 \\
\hline $\begin{array}{l}\text { 3-phase modified } \\
\text { EMT model }\end{array}$ & 41.2 & 10.3 & 18.2 & 243.8 & 35.7 \\
\hline
\end{tabular}

Tab. 1: Elastic constants of T-300 carbon fibre derived from different experimental works and theoretical calculations. 


\section{Deduction of C-fibre elastic constants from nanoindentation}

Nanoindentation was carried out on transversal (Location A) and longitudinal (Location B) cross-sections of C-fibres as is shown in Fig. 3.
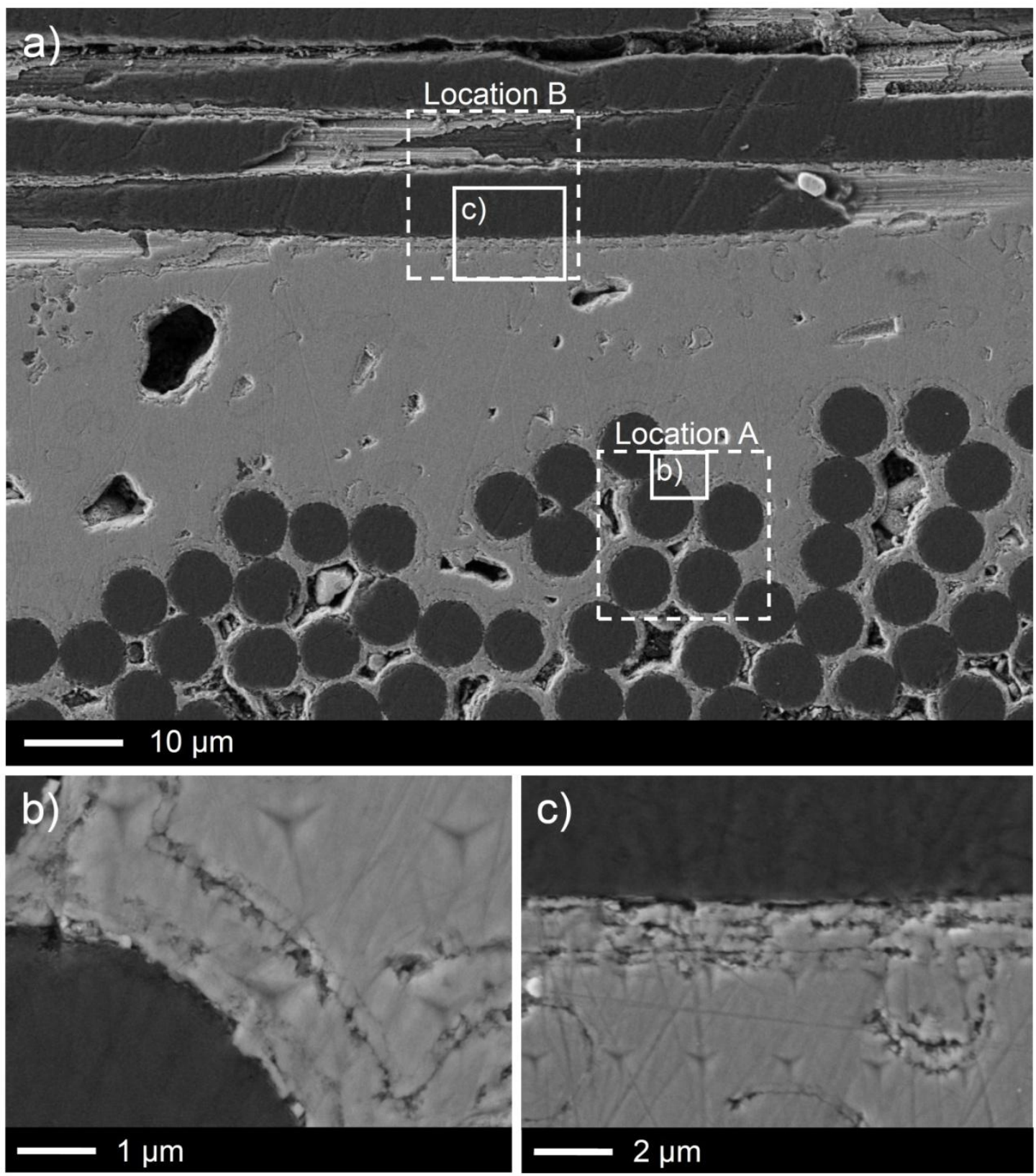

Fig. 3: a) Locations of indents on the microstructure of $2 \mathrm{D}-\mathrm{C} / \mathrm{SiC}$ composite and their arrangement on b) transversal and c) longitudinal cross-sections of fibres. 
Indents, arranged in arrays of 10x10, covered several fibres of both orientations and also the silicon carbide phase. These SEM images suggest that the deformation of fibres is mostly elastic as the residual imprints are not discernible. Contrary to this, indents are visible in silicon carbide phase due to a considerable plasticity. These observations are confirmed by the characteristic load-displacement curves indicated in Fig. 4a. Despite SEM images which suggest elastic deformation of C-fibres, only the longitudinal cross-section behaves purely elastically during indentation but the transversal cross-section shows a slight plasticity.
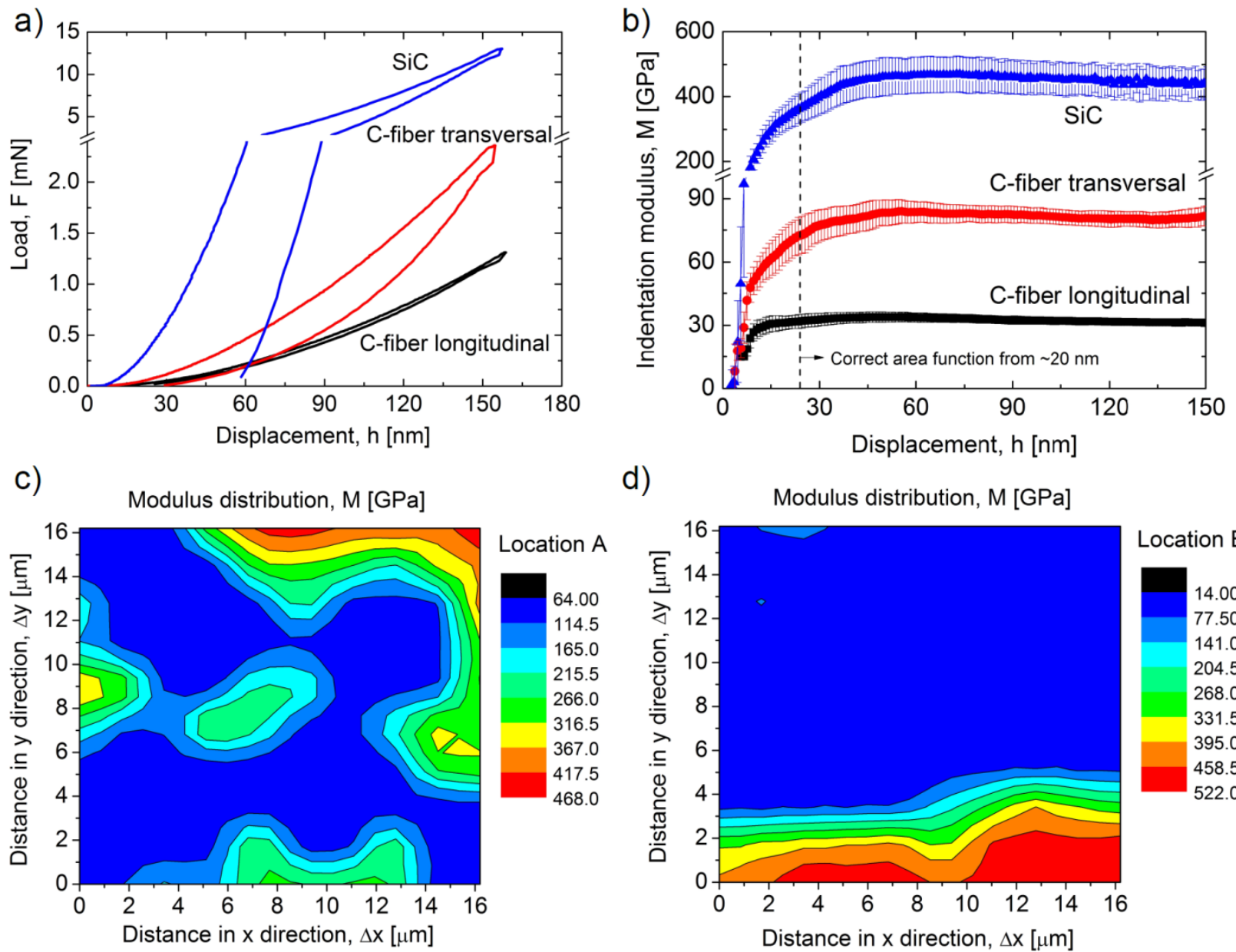

d)
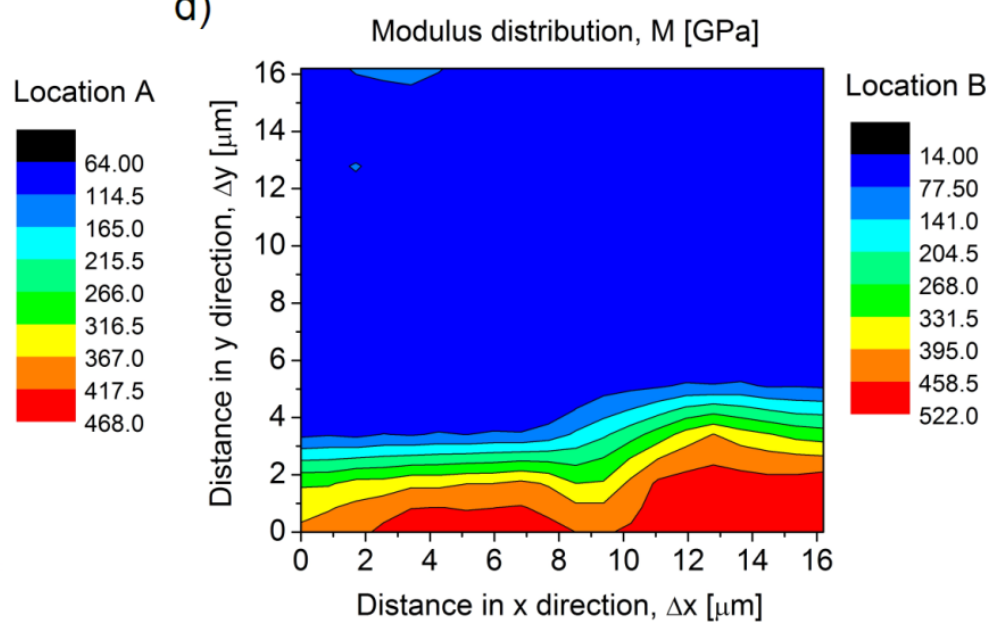

Fig. 4: a) Characteristic indentation load-displacement curves of transversal, longitudinal crosssections of individual carbon fibres and $\mathrm{SiC}$ matrix. b) Averaged indentation modulus-depth profiles corresponding to each constituent and their 2D maps at c) Location A and d) Location B. 
Figure 4a shows also that the transversal cross-section requires about twice as high a load as the longitudinal one to generate the same penetration depth. This anisotropy can be observed on the averaged indentation modulus depth profiles shown in Fig. $\mathbf{4 b}$. Only those indents were averaged which were located entirely inside each fibre cross-section and SiC phase. The averaged indentation modulus depth profiles show constant values over $\sim 20 \mathrm{~nm}$ where the area function used is correct. Here, only the modulus curves are indicated as the focus is on the elastic behaviour of the fibres but the measured hardness profiles show similar tendencies above $\sim 60$ nm. Quantitatively, the measured indentation modulus $(M)$ and hardness $(H)$ of fibres are obtained to $M_{T-\text { section }}=81.6 \pm 4.6 \mathrm{GPa}, H_{T \text {-section }}=6.8 \pm 0.4 \mathrm{GPa}$ and $M_{L \text {-section }}=32.5 \pm 1.9 \mathrm{GPa}, H_{L \text { - }}$ section $=4.7 \pm 0.4 \mathrm{GPa}$ corresponding to the transversal and longitudinal cross-sections, respectively. The $M$ values of single tests, corresponding to the depth of $150 \mathrm{~nm}$, are plotted in the form of 2D indentation maps for both locations in Figs. 4c and 4d. These maps show similar patters as the SEM image in Fig. 3a indicating the accuracy of nanoindentation. The measured anisotropy in the indentation modulus is in agreement with a recent nanoindentation report on carbon fibres [19]. Considering the hardness anisotropy and elasticity of fibres, our results sufficiently differ from that nanoindentation work which is most probably attributed to different fibre structure and the higher applied loads.

To analyze the effect of indenter geometry and the influence of SiC matrix on indentation, FEM calculations were performed on the transversal and longitudinal cross-section of a model Cfibres. Indentations were simulated by conical and Berkovich tip geometries and the elastic properties of filament were selected similar to T-300 fibre [7]. The obtained $M$ values are indicated in Tab. 2. 


\begin{tabular}{ccc}
\hline \multirow{2}{*}{ Cross-section of fibre } & \multicolumn{2}{c}{ Indentation modulus, $M[\mathrm{GPa}]$} \\
\cline { 2 - 3 } & conical tip, FEM & Berkovich tip, FEM \\
\hline transversal & 99.01 & 106.34 \\
longitudinal & 25.89 & 27.86 \\
\hline
\end{tabular}

Tab. 2: Simulated indentation modulus values for exact transversal and longitudinal crosssections of carbon fibre by FEM.

The ratio of FEM simulated indentation modulus using Berkovich and conical tips were 1.074 and 1.069 for transversal and longitudinal cross-sections, respectively. This suggests that the $M$ values are $\sim 7 \%$ lower for conical tips than what corresponds to the Berkovich tip. This result is in a good agreement with the calculations performed by Vlassak and Nix [24]. The simulated stress fields under the Berkovich indents at a maximum depth of $150 \mathrm{~nm}$ for both cross-sections are shown in Fig. 5. This graph shows that the generated stress fields practically vanish at the boundary of the fibre, indicating that the surrounding hard $\mathrm{SiC}$ matrix has no influence on the measured indentation modulus. It confirms that the measured values, indicated in Fig. 4, really correspond to the material properties at the location where the indents are. Figure 5 shows also that the shape of the stress fields is fairly different due to the fibre anisotropy. On a transversal cross-section, the stress field penetrates along the fibre axis while it is more shallow and extended perpendicular to the indentation direction on the longitudinal cross-section. 

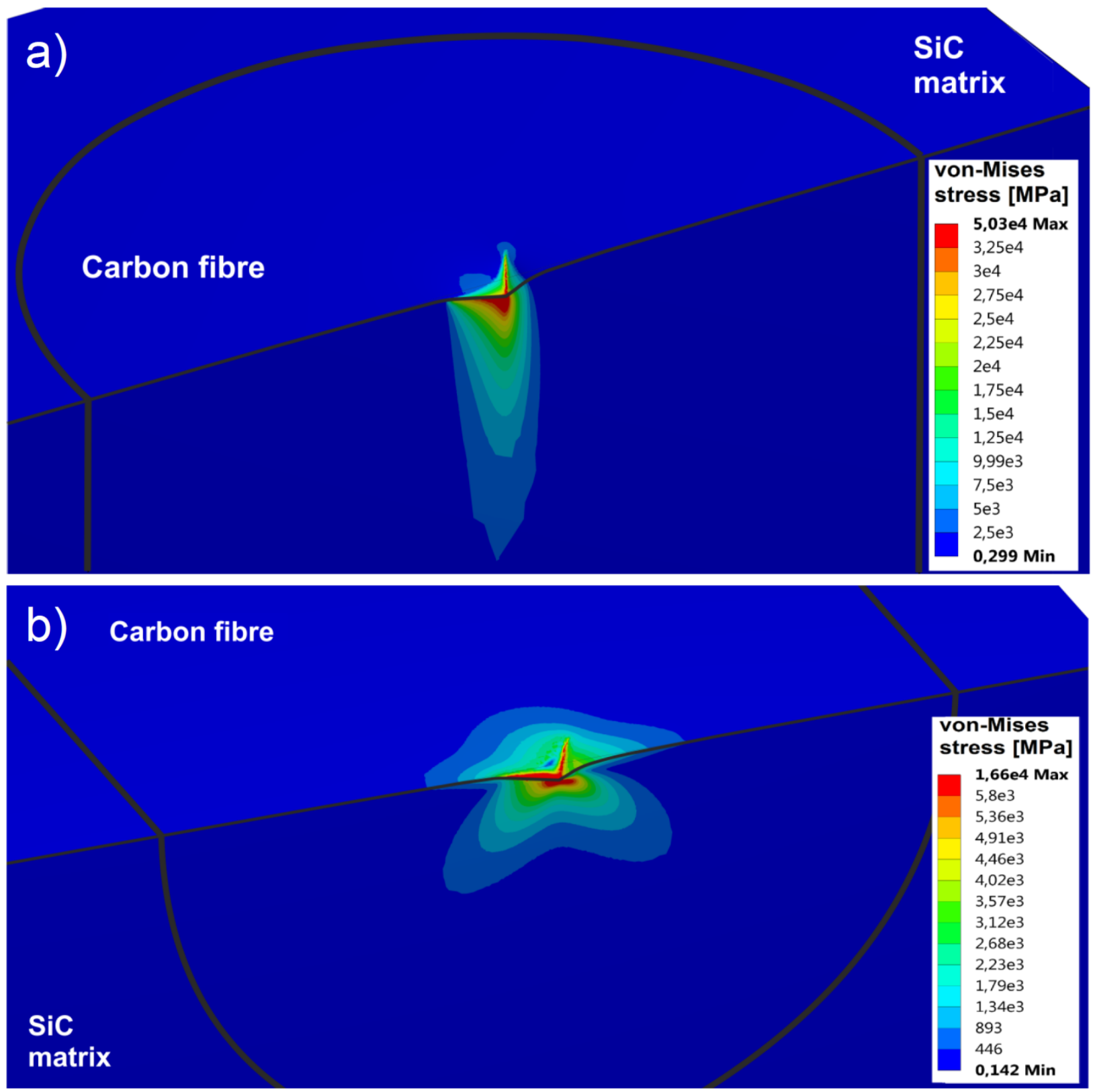

Fig. 5: Stress fields under the Berkovich indents simulated by FEM in a) transversal and b)

longitudinal cross-sections of carbon fibres.

The orientation dependence of C-fibre was also analyzed by FEM and the Vlassak-Nix model. Results show that the indentation modulus decreases with an increasing tilt angle $(\alpha)$ with respect to the exact longitudinal direction (direction parallel with the fibre axis). The reduction of 
$M$ is significant for low tilt angle, it decreases to half up to $\alpha \sim 35^{\circ}$, then the decrease moderates and becomes approximately constant over $\alpha \sim 75^{\circ}$. The shape of the curve is similar to the graph shown in Fig. 7 which is discussed later. The orientation dependence obtained suggests that the inclination of carbon fibre with respect to its longitudinal direction has a considerable influence on the evaluation of nanoindentation data. Therefore, the shape of fibres assumed to be transversal cross-section were analyzed by SEM and AFM as it is shown in Fig. 6.

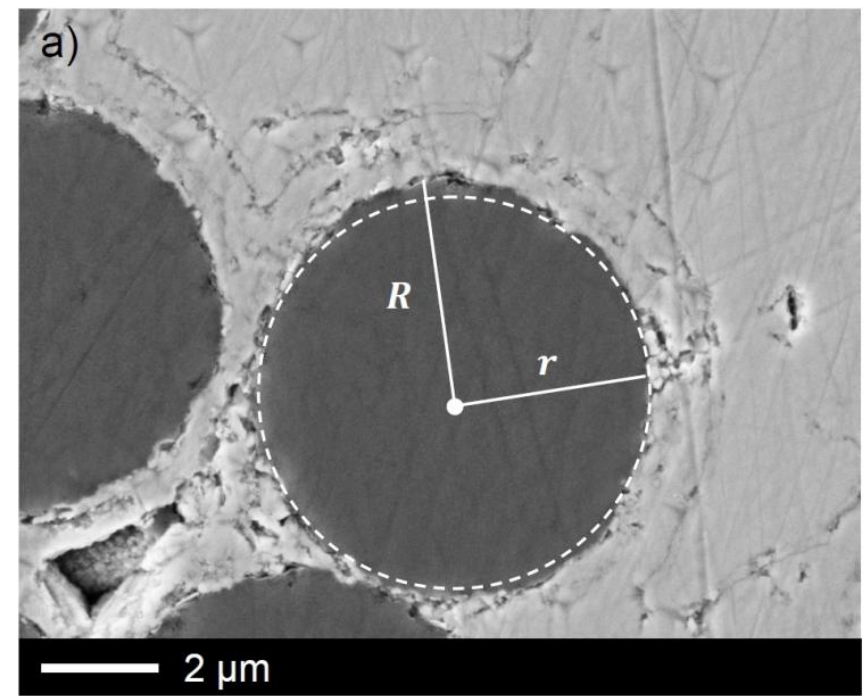

b)
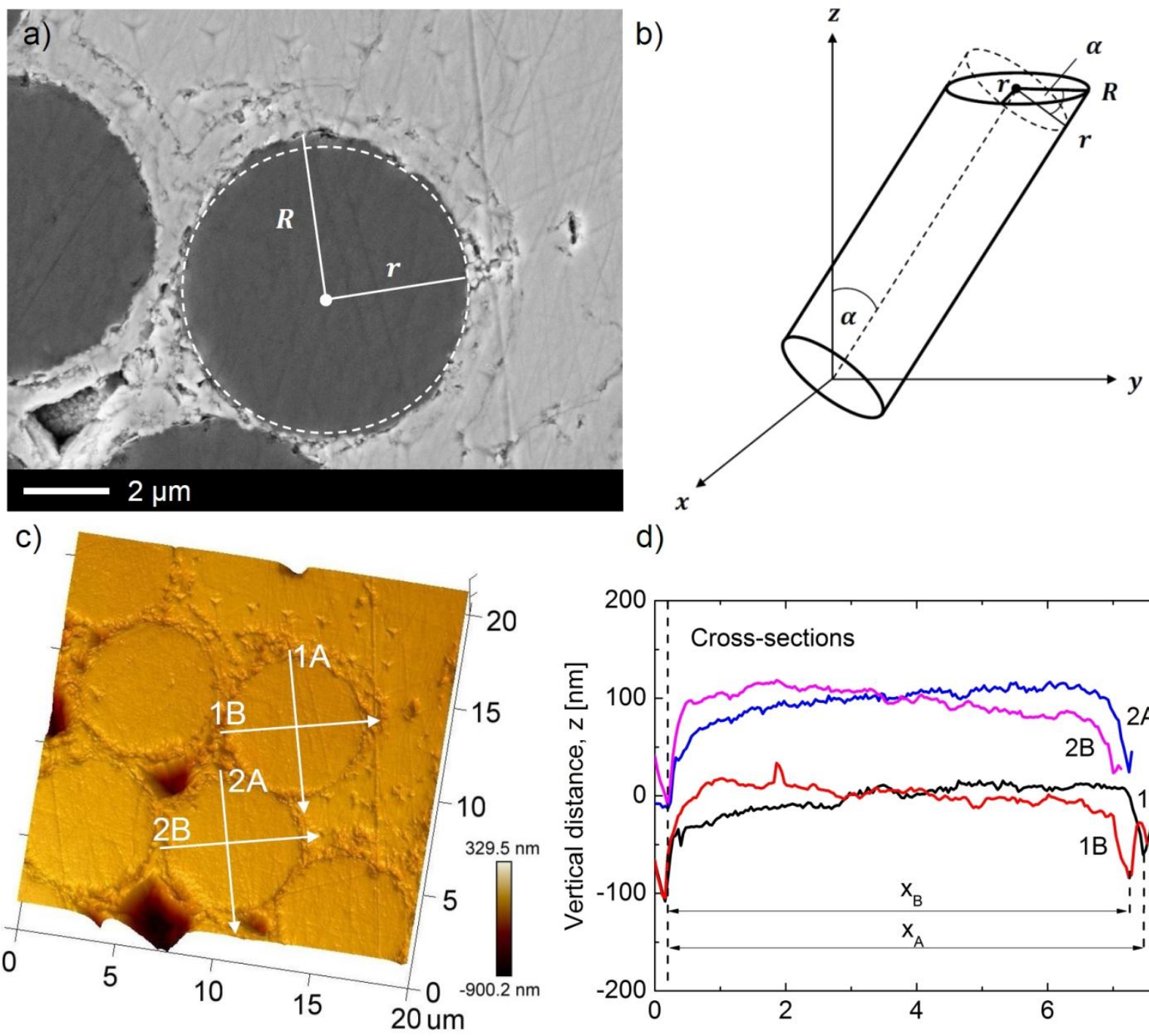

d)

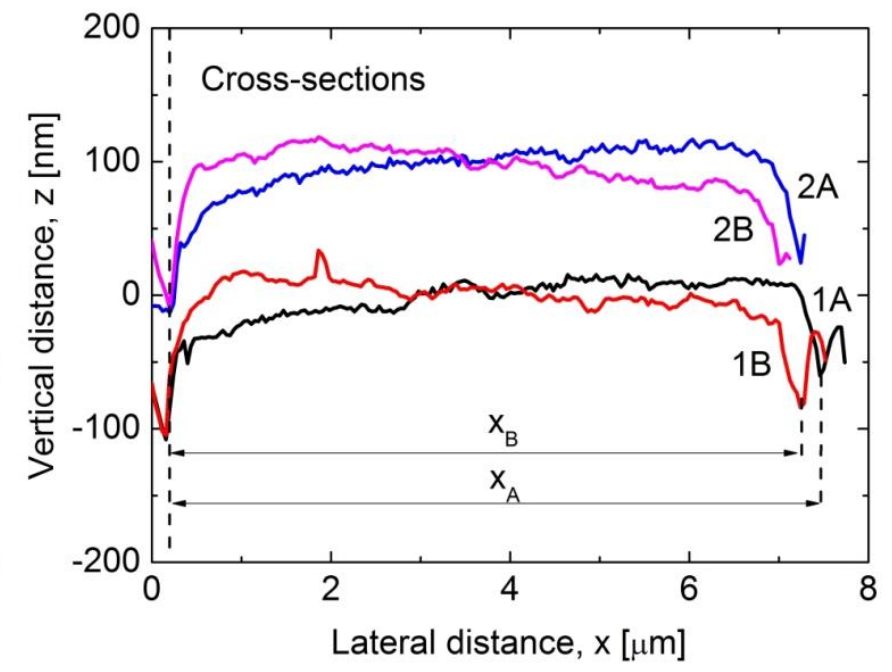


Fig. 6: The cross-sectional shape of carbon fibres: a) SEM, c) AFM. The deviation from the ideal circular shape is caused by b) the inclination of the fibre which is correlated to d) the major and minor diameters of ellipsoids measured by AFM.

A small deviation of fibre orientation from the ideal longitudinal cross-section (indentation in transversal direction) has practically no effect on the indentation modulus as mentioned before. Figure 6 shows that the shape of transversal cross-sections is not circular but has a slightly ellipsoidal shape. The major and minor radii are dented by $R$ and $r$, respectively (Fig. 6a). Using these values, the tilt angle of fibres $(\alpha)$ can be expressed by a simple relationship according to Fig. 6 b.

$$
\alpha=\cos ^{-1}\left(\frac{r}{R}\right)
$$

The ratio of $r$ and $R$ were calculated by AFM measuring the major $\left(x_{A}\right)$ and minor $\left(x_{B}\right)$ diameters of the fibres shown in Figs. 6c,d. This resulted in an average tilt angle of $13.9^{\circ} \pm 1.3^{\circ}$ for fibres assumed to have transversal cross-section. The measured inclination is probably attributed to the wavy shape of fibres in their plain weave structure.

Taking into account the FEM and AFM results, the measured indentation modulus values were converted to their equivalent values for conical indenter and associated with the proper tilt angles $\left(14^{\circ}\right.$ and transversal direction). The obtained values are $M_{14^{\circ}}=75.9 \pm 4.3 \mathrm{GPa}$ and $M_{T}=30.2 \pm 1.8 \mathrm{GPa}$. To deduce the elastic constants of C-fibre by Eqs. (3)-(7), the indentation modulus value of the longitudinal direction is required. This value is slightly higher than $M_{14^{\circ}}$ and related to that as follows.

$$
M_{L}=x \cdot M_{14^{\circ}}
$$


Here, the coefficient of $x$ is selected to be in the range of 1.0-1.3 based on the earlier simulations of orientation dependence of $M$. Fixing the $x$ value, the five components of fibre stiffness tensor were calculated analytically using Eqs. (3)-(7) and (14). During calculation, the longitudinal Young's modulus of T-300 fibre is selected to $E_{L}=230 \mathrm{GPa}$ reported by the manufacturer. Based on the obtained elastic constants, the indentation modulus for the tilt angle of $14^{\circ}$ was calculated numerically by the Vlassak-Nix method. Iteratively, the $x$ value was varied until the calculated indention modulus approximated the measured $M_{14^{\circ}}$ within the experimental scatter of $5 \%$. The deduced elastic constants are indicated in Tab. 1 and the orientation dependence of $M$ is shown in

Fig. 7.

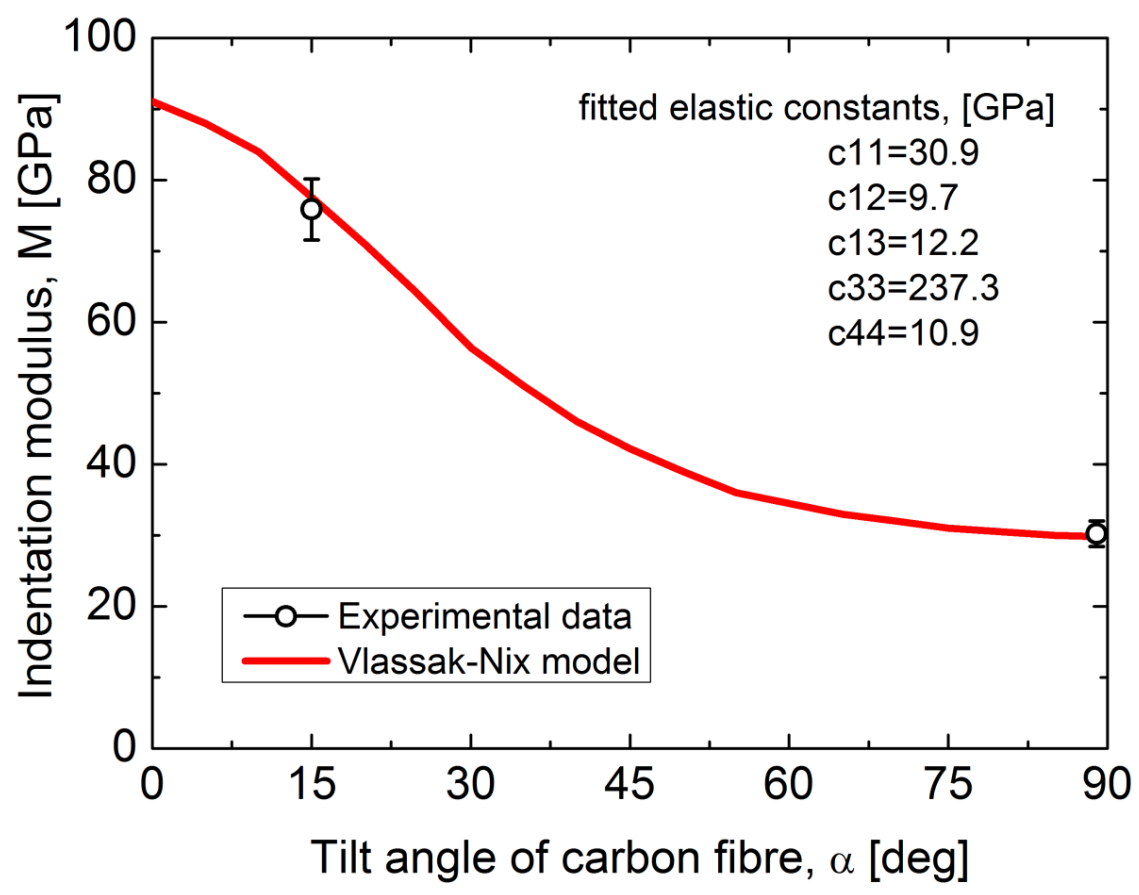

Fig. 7: Orientation dependence of the indentation modulus of T-300 carbon fibre calculated by the Vlassak-Nix model. The used elastic constants were derived from the proposed nanoindentation based method. 
The points of the red curve were calculated by a step size of $5^{\circ}$. This plot shows that inclination of fibre has a significant effect as the measured $M_{14^{\circ}}$ is about $20 \%$ lower than the indentation modulus in an exact longitudinal direction $\left(M_{L}\right)$. The obtained elastic constants are in good agreement with ultrasound measurements of similar PAN based fibres with the same longitudinal Young's modulus and from the same manufacturer [7] shown in Tab. 1. It suggests that the heat treatment which the fibres were subjected to during sintering has a minor effect on elastic properties in agreement with recent reports $[12,13]$. The sensitivity analysis revealed that effect of Poisson's ratios $\left(v_{T T}, v_{L T}\right)$, selected from the range of $0.1-0.4$, is negligible on the deduced elastic constants. The influence of longitudinal modulus $\left(E_{L}\right)$ of fibre has an impact mostly on the shear modulus $\left(c_{44}\right), 10 \%$ change of $E_{L}$ causes a $\sim 10 \%$ difference in $c_{44}$. Taking into account that the longitudinal elastic modulus and Poisson's ratios were predetermined, this nanoindentation method supplies an independent and accurate measurement of transversal elastic modulus $\left(E_{T}\right)$ and shear modulus in the longitudinal plane $(G)$ of carbon fibres. Shear modulus is equal to $c_{44}$ constant as $G=c_{44}$, while the expression for $E_{T}$ is as follows.

$$
E_{T}=\frac{\left(c_{11}-c_{12}\right) \cdot\left(c_{11} \cdot c_{33}+c_{12} \cdot c_{33}-2 \cdot c_{13}^{2}\right)}{c_{11} \cdot c_{33}-c_{13}^{2}}
$$

Transversal elastic modulus is calculated to $27.6 \mathrm{GPa}$ and is in good agreement with FEM simulation and theoretical calculations reported for T-300 fibres by Miyagawa et al. [15]. It is important to note that the indentation modulus measured along the transversal direction (perpendicular to the longitudinal cross-section, $M_{L-\text { section }}=32.5 \pm 1.9 \mathrm{GPa}$ ) is quite close to the transversal Young's modulus, unlike that paper [15]. Moreover, the indentation modulus measured in the longitudinal direction is fairly different from the corresponding elastic modulus, and cannot be identified with it for such highly anisotropic materials such as carbon fibres. This 
indicates that nanoindentation is a powerful tool to measure elastic properties of carbon fibres but it requires an appropriate evaluation method as we have proposed.

\section{Nanostructural based prediction of carbon fibre elasticity}

In this section, the elastic constants of T-300 carbon fibre are predicted and compared with the experimental values using the previously described Eshelby-Mori-Tanaka (EMT) model for 2 and 3 phase cases. In brief, the models require the aspect ratio of ellipsoidal crystals $\left(t_{c}=L_{c} / L_{a}\right)$ and microvoids $\left(t_{v}=L / a\right)$, the orientation parameter of crystals $(H)$, the volume fraction of crystalline carbon $\left(f_{c}\right)$ and voids $\left(f_{v}\right)$, and the elastic constants of each phase $\left(C_{m}, C_{c 0}, C_{v 0}\right)$. All these parameters are known from the literature measured by TEM, x-ray and ultrasound [14, 21].

During 2-phase EMT model calculation, the volume fraction of crystalline carbon was set equal to the apparent crystallinity of T-300 fibre as $f_{c}=0.6836$ measured by X-ray [21]. The orientation parameter and the aspect ratio of oblate shape crystals were $H=0.3626$ and $t_{c}=0.453$, respectively, based on XRD measurements. These values are in good agreement with other papers reported in the literature [5, 13]. Elastic constants are defined in Appendix B where the Young's modulus of the matrix was selected to $E_{m}=137.98 \mathrm{GPa}$ according to [21] (Poisson's ratio is fixed, $\left.v_{m}=0.3\right)$. The calculated stiffness tensor of carbon fibre is practically transversely isotropic; a small difference between $c_{13}$ and $c_{31}$ components $(\sim 7 \%)$ is caused by the nonsymmetrical shape of the Eshelby tensor. The obtained fibre elastic constants were far enough from the experimental results. In the literature $[14,21]$, this problem is commonly solved by changing either the Young's modulus of matrix or the crystalline volume fraction to get a good fit with the experimental longitudinal modulus of the fibre $\left(E_{L}=230 \mathrm{GPa}\right)$. This was carried out here either reducing the elastic modulus to $E_{m}=69 \mathrm{GPa}$ or the crystalline phase fraction to $f_{c}=0.36$. 
The obtained elastic constants of the fibre are indicated in Tab. 1. The aspect ratio of crystals has a slight influence on calculation and the orientation parameter was kept fixed as it is quite characteristic for T-300 fibre. Despite the good fit in $E_{L}$, the predicted constants are still far enough from the experimental results. This is caused by the nature of the isotropic stiffness tensor of the matrix which has relative high off-diagonal elements and shear components. A good fit may be achieved for the shear component $\left(c_{44}\right)$ without applying an orientation average around the fibre axis, as was reported by Tanaka et al. [14]. In this case, the calculated stiffness tensor is rather different form the transversely isotropic shape due to the nearly aligned graphitic structure.

Similar calculations were carried out applying the 3-phase EMT model. Here, the used parameters were extended with the aspect ratio and the volume fraction of microvoids, respectively $t_{v}=2.716$ and $f_{v}=0.0973$, which were measured by SAXS [21]. The results were far enough from the experiment`s similarly to the 2-phase case. Good fit with the longitudinal elastic modulus was achieved by reducing either the matrix modulus to $E_{m}=81 \mathrm{GPa}$ or the volume fraction of crystalline phase to $f_{c}=0.44$. The calculated elastic constants for a 3 -phase case are shown in Tab. 1. Taking into account the presence of microvoids (3-phase model), better approximation is found in for the fibre elastic properties using reduced matrix modulus than in 2phase case. However, the predictions of both 2 and 3-phase EMT models are still not in agreement with the experiments. The analysis of simulated elastic constants revealed that the ratio of $c_{33}$ to shear component $\left(c_{44}\right)$ is around 3.5-3.8 which coincides with the ratio of corresponding matrix components $\left(C_{m}\right)$. These findings and the relatively high shear component ( $\left.c_{44}\right)$ suggest that probably better agreement could be achieved with the experiments by changing the stiffness tensor of amorphous matrix. A physically reasonable form of carbon which possesses high longitudinal modulus with low shear component is the graphite aligned with its planes parallel with the fibre axis. The stiffness components of this aligned graphite, with 
randomly oriented symmetry axis in the transverse plane of the fibre, were calculated with the 2phase EMT model. Here, only the volume fraction and orientation parameter of crystals were changed to $f_{c}=1$ and $H=0$, respectively. The calculated stiffness tensor of aligned graphite was not symmetrical, thus the $c_{13}, c_{23}$ components were substituted by $c_{31}$. The obtained transversely isotropic tensor $\left(C_{c-a l i g n e d}\right)$ is indicated in Appendix B.

In order to improve the prediction power of the Eshelby-Mori-Tanaka approximation, a modified EMT model was proposed. A new anisotropic stiffness was defined for the 'amorphous' matrix composed of aligned graphite planes parallel with the fibre axis $\left(C_{c \text {-aligned }}\right)$ and voids $\left(C_{v}\right)$ among them. The stiffness tensor of the new matrix was defined according to the rule of mixture as follows $\left(C_{v}\right.$ is identical with the null tensor).

$$
C_{m}^{\text {new }}=\gamma \cdot C_{c-\text { aligned }}+(1-\gamma) \cdot C_{v}=\gamma \cdot C_{c-\text { aligned }}
$$

Here, $\gamma$ represents the volume ratio of aligned graphite in the amorphous matrix. The rest of the parameters were identical with that defined earlier. Simulations were performed for 2 and 3phase cases using the present modified EMT model. All the parameters were fixed to their initial values, including $E_{m}$ and $f_{c}$, the only fitting parameter was $\gamma$ which was used to adjust the longitudinal elastic modulus to $E_{L}=230 \mathrm{GPa}$. Appropriate fits were obtained using the values of $\gamma=0.215$ and $\gamma=0.3$ for 2 and 3 -phase cases, respectively. The calculated fibre elastic constants are indicated in Tab. 1. The predictions of modified EMT models are quite close to each other and are much closer to the experimental results compared to the earlier models. Despite the results of the proposed modified approach being slightly higher than the experimental values, the predicted $c_{11}$ and $c_{44}$ constants are less than the half of that which was reached by the conventional micromechanical models. This shows that the elasticity of the matrix has a significant effect on the predicted fibre properties. Additionally, it suggests that the amorphous 
carbon matrix has anisotropic elastic properties rather than an isotropic form. It is important to note that the present model was developed for the T-300 fibres, but it can be applied for any type of carbon fibres possessing transversely isotropic symmetry. Beside that the present work focused on the T-300 fibre, the reason why the model was not tested on other fibres is the lack of reliable elastic constants with the corresponding nanostructural parameters in the literature. Despite that, using different nanostructural parameters, a wide range of longitudinal modulus values $\left(E_{L}=100\right.$ $900 \mathrm{GPa})$ with relatively low shear components $\left(c_{44}=20-50 \mathrm{GPa}\right)$ can be successfully predicted by the present model.

\section{Conclusions}

A nanoindentation based method was developed to measure directly the elastic constants of carbon fibres embedded in composite systems. The evaluation method allows one to measure independently two of the five constants of transversely isotropic fibres, while the other three are derived from other sources. Although nanoindentation cannot be considered as an alternative of the ultrasound technique as itself, it may be completed using additional measurements (e.g. tensile test, X-ray, etc.) to deduce the stiffness components of C-fibres. In this work, a case study was carried out on a $2 \mathrm{D}-\mathrm{C} / \mathrm{SiC}$ composite system and the elastic constants were determined for the contained T-300 carbon fibres. The obtained results are in good agreement with ultrasound measurements reported in the literature. Here, it is important to emphasize that the proposed method is valid for any kind of fibres possessing transversely isotropic symmetry. In order to predict the experimental elastic constants, two- and three-phase Eshelby-Mori-Tanaka (EMT) micromechanical models were applied based on the nanostructure of T-300 fibre. During calculations, fibre structure was assumed to be composed of amorphous and crystalline carbon 
(two-phase model) and amorphous matrix, crystalline carbon and microvoids (three-phase model) using nanostructural parameters available in the literature determined by X-ray. Although the elastic properties of carbon fibres had been already simulated by similar micromechanical models, in this work, for the first time the complete set of elastic constants were analyzed. Simulations revealed that a good fit cannot be achieved with the measured stiffness components despite the agreement with the longitudinal Young's modulus. Analysis of the calculated constants suggested that the breakdown of the conventional EMT model is attributed to the assumed isotropy of amorphous matrix. To improve the prediction power of the micromechanical models used, a new transversely isotropic matrix was proposed for the 'amorphous' phase. This is considered as a mixture of aligned graphite planes and voids among them. The volume ratio of aligned graphite in the matrix was determined as the only fitting parameter of the proposed modified micromechanical model. The predicted elastic constants are slightly higher than the experimental values, but are in much better agreement with them than that which was reached by earlier models. It is important to note that the present model was developed for the T-300 fibres but can be applied for any type of carbon fibres possessing transversely isotropic symmetry. Additionally, the elastic properties of carbon fibres could be improved further using the proposed measuring and modelling methods.

\section{Acknowledgements}

The work was supported by the Slovak Grant Agency for Science under the projects: APVV-15-0469 and VEGA 2/0163/16. The preparation of the composite was supported by the National Science Foundation of China (No. 51572224) and the 111 Project (No. B08040). The 
authors would like to thank A. Kovalčíková (IMR-SAS, Košice) for SEM investigation. The support of Keysight Technologies is greatly acknowledged.

\section{Appendix A. Indentation modulus by Vlassak-Nix model}

The formula for the calculation of indentation modulus based on the Vlassak-Nix model [24] is as follows.

$$
\begin{gathered}
M=16 \cdot \pi^{2} \cdot\left(\int_{0}^{2 \pi} \alpha_{m} \cdot B_{k m}^{-1}(\gamma) \cdot \alpha_{k} d \gamma\right)^{-1} \\
B_{j s}(\underline{t})=\left(8 \cdot \pi^{2}\right)^{-1} \cdot \int_{0}^{2 \pi}(\underline{m} \underline{m})_{j s}-(\underline{m} \underline{n})_{j k} \cdot(\underline{n} \underline{n})_{k r}^{-1} \cdot(\underline{n} \underline{m})_{r s} d \varphi \\
(\underline{a} \underline{b})_{j k}=\underline{a}_{i} \cdot C_{i j k l} \cdot \underline{b}_{l}
\end{gathered}
$$

In Eq. (A1), $\alpha_{\mathrm{i}}$ denotes the direction cosines of tilt angles from the indentation direction towards the $x_{1}, x_{2}$ and $x_{3}$ axes of the fibre. The coordinate system in which the calculation of $M$ was carried out is identical with the fibre coordinate system where $x_{3}$ is parallel with its symmetry axis. Furthermore $(\underline{m}, \underline{n}, \underline{t})$ form a right hand coordinate system, where $\underline{t}$ lies in the plane perpendicular to the indentation direction. In Eq. (A3), the repeated indices imply a summation

over the repeated index from 1 to 3 . The components of $C_{i j k l}$ are expressed by the elements of its contracted (Voigt) tensor $\left(c_{i j}\right)$ using the relationship between the terms ( $i j$ or $\left.k l\right)$ of $C_{i j k m}$ and single numbers $(m$ or $n)$ of $c_{m n}$ tensors which is $(i j$ or $k l) \rightarrow(m$ or $n)$, i.e. $11 \rightarrow 1,22 \rightarrow 2,33 \rightarrow 3,23,32 \rightarrow 4$, $31,13 \rightarrow 5$ and $12,21 \rightarrow 6$. Further details can be found elsewhere [24, 25].

\section{Appendix B. Form of stiffness and Eshelby tensors}


In the theory of linear elasticity, the relationship between the stress $(\sigma)$ and strain $(\varepsilon)$ fields is determined by the generalized Hook's law as follows.

$$
\sigma=\left(\begin{array}{lll}
\sigma_{11} & \sigma_{12} & \sigma_{13} \\
\sigma_{12} & \sigma_{22} & \sigma_{23} \\
\sigma_{13} & \sigma_{23} & \sigma_{33}
\end{array}\right)=C \cdot \varepsilon=C_{i j k l} \cdot\left(\begin{array}{lll}
\varepsilon_{11} & \varepsilon_{12} & \varepsilon_{13} \\
\varepsilon_{12} & \varepsilon_{22} & \varepsilon_{23} \\
\varepsilon_{13} & \varepsilon_{23} & \varepsilon_{33}
\end{array}\right)
$$

Here, the $C_{i j k l}$ is the fourth order stiffness tensor, $\sigma$ and $\varepsilon$ are the symmetrical stress and strain tensors, respectively. Using the contracted Voigt notation, Eq. (B1) has the below form:

$$
\left(\begin{array}{l}
\sigma_{11} \\
\sigma_{22} \\
\sigma_{33} \\
\sigma_{23} \\
\sigma_{13} \\
\sigma_{12}
\end{array}\right)=C_{i j} \cdot\left(\begin{array}{c}
\varepsilon_{11} \\
\varepsilon_{22} \\
\varepsilon_{33} \\
2 \cdot \varepsilon_{23} \\
2 \cdot \varepsilon_{13} \\
2 \cdot \varepsilon_{11}
\end{array}\right)
$$

where $C_{i j}$ represents the symmetrical stiffness tensor in a form of a $6 x 6$ matrix. Definition of stiffness tensors of amorphous matrix $\left(C_{m}\right)$, crystalline carbon $\left(C_{c 0}\right)$ and mirovoids $\left(C_{v 0}\right)$, indicated in Eqs. (9), (11) and (12), are shown below. The isotropic matrix is defined by the Lamé constants $(\lambda, \mu)$ which can be expressed by the Young's modulus $\left(E_{m}\right)$ and Poisson's ratio $\left(v_{m}\right)$.

$$
\begin{gathered}
\lambda=\frac{-v_{m} \cdot E_{m}}{\left(2 \cdot v_{m}-1\right) \cdot\left(1+v_{m}\right)}, \mu=\frac{E_{m}}{2 \cdot\left(1+v_{m}\right)} \\
C_{m}=\left(\begin{array}{cccccc}
2 \cdot \mu+\lambda & \lambda & \lambda & 0 & 0 & 0 \\
\lambda & 2 \cdot \mu+\lambda & \lambda & 0 & 0 & 0 \\
\lambda & \lambda & 2 \cdot \mu+\lambda & 0 & 0 & 0 \\
0 & 0 & 0 & \mu & 0 & 0 \\
0 & 0 & 0 & 0 & \mu & 0 \\
0 & 0 & 0 & 0 & 0 & \mu
\end{array}\right)
\end{gathered}
$$

The transversely isotropic crystalline carbon is defined by the elastic constants of graphite $c_{11}=1060 \mathrm{GPa}, c_{12}=180 \mathrm{GPa}, c_{13}=15 \mathrm{GPa}, c_{33}=36.5 \mathrm{GPa}$ and $c_{44}=4 \mathrm{GPa}$ [29]. 


$$
C_{c 0}=\left(\begin{array}{cccccc}
c_{11} & c_{12} & c_{13} & 0 & 0 & 0 \\
c_{12} & c_{11} & c_{13} & 0 & 0 & 0 \\
c_{13} & c_{13} & c_{33} & 0 & 0 & 0 \\
0 & 0 & 0 & c_{44} & 0 & 0 \\
0 & 0 & 0 & 0 & c_{44} & 0 \\
0 & 0 & 0 & 0 & 0 & \frac{c_{11}-c_{12}}{2}
\end{array}\right)
$$

Stiffness tensor of microvoid is identical with the null tensor.

$$
C_{v 0}=\left(\begin{array}{cccccc}
0 & 0 & 0 & 0 & 0 & 0 \\
0 & 0 & 0 & 0 & 0 & 0 \\
0 & 0 & 0 & 0 & 0 & 0 \\
0 & 0 & 0 & 0 & 0 & 0 \\
0 & 0 & 0 & 0 & 0 & 0 \\
0 & 0 & 0 & 0 & 0 & 0
\end{array}\right)
$$

Using the contracted Voigt notation, the components of Eshelby tensors $\left(E_{c}, E_{v}\right)$ are as follows [30].

$$
\begin{aligned}
& E_{c, v}=\left(\begin{array}{cccccc}
E_{11} & E_{12} & E_{13} & 0 & 0 & 0 \\
E_{12} & E_{11} & E_{13} & 0 & 0 & 0 \\
E_{31} & E_{31} & E_{33} & 0 & 0 & 0 \\
0 & 0 & 0 & E_{44} & 0 & 0 \\
0 & 0 & 0 & 0 & E_{44} & 0 \\
0 & 0 & 0 & 0 & 0 & E_{66}
\end{array}\right) \\
& E_{11}=\frac{3}{8 \cdot\left(1-v_{m}\right)} \cdot \frac{t^{2}}{t^{2}-1}+\frac{1}{4 \cdot\left(1-v_{m}\right)} \cdot\left(1-2 \cdot v_{m}-\frac{9}{4 \cdot\left(t^{2}-1\right)}\right) \cdot g \\
& E_{12}=\frac{1}{4 \cdot\left(1-v_{m}\right)} \cdot\left(\frac{t^{2}}{2 \cdot\left(t^{2}-1\right)}-\left(1-2 \cdot v_{m}+\frac{3}{4 \cdot\left(t^{2}-1\right)}\right) \cdot g\right) \\
& E_{13}=-\frac{1}{2 \cdot\left(1-v_{m}\right)} \cdot \frac{t^{2}}{t^{2}-1}+\frac{1}{4 \cdot\left(1-v_{m}\right)} \cdot\left(\frac{3 \cdot t^{2}}{t^{2}-1}-\left(1-2 \cdot v_{m}\right)\right) \cdot g \\
& E_{31}=-\frac{1}{2 \cdot\left(1-v_{m}\right)} \cdot\left(1-2 \cdot v_{m}+\frac{1}{t^{2}-1}\right)+\frac{1}{2 \cdot\left(1-v_{m}\right)} \cdot\left(1-2 \cdot v_{m}+\frac{3}{2 \cdot\left(t^{2}-1\right)}\right) \cdot g \\
& E_{33}=\frac{1}{2 \cdot\left(1-v_{m}\right)} \cdot\left(1-2 \cdot v_{m}+\frac{3 \cdot t^{2}-1}{t^{2}-1}-\left(1-2 \cdot v_{m}+\frac{3 \cdot t^{2}}{t^{2}-1}\right) \cdot g\right) \\
& E_{44}=\frac{1}{4 \cdot\left(1-v_{m}\right)} \cdot\left(1-2 \cdot v_{m}-\frac{t^{2}+1}{t^{2}-1}-\frac{1}{2} \cdot\left(1-2 \cdot v_{m}-\frac{3 \cdot\left(t^{2}+1\right)}{t^{2}-1}\right) \cdot g\right) \\
& E_{66}=\frac{1}{4 \cdot\left(1-v_{m}\right)} \cdot\left(\frac{t^{2}}{2 \cdot\left(t^{2}-1\right)}+\left(1-2 \cdot v_{m}-\frac{3}{4 \cdot\left(t^{2}-1\right)}\right) \cdot g\right)
\end{aligned}
$$


where $v_{m}$ is the Poisson's ratio of matrix, $t$ is the aspect ratio of crystals and inclusions $\left(t_{c}=L_{c} / L_{a}\right.$, $\left.t_{v}=L / a\right)$ and $g$ is given by

$$
\begin{aligned}
& g_{c}=\frac{t}{\left(1-t^{2}\right)^{3 / 2}} \cdot\left(\cos ^{-1} t-t \cdot\left(1-t^{2}\right)^{\frac{1}{2}}\right) \\
& g_{v}=\frac{t}{\left(t^{2}-1\right)^{3 / 2}} \cdot\left(t \cdot\left(t^{2}-1\right)^{\frac{1}{2}}-\cosh ^{-1} t\right)
\end{aligned}
$$

Stiffness tensor of the aligned graphite is calculated by EMT model as follows, components are in GPa.

$$
C_{\text {c-aligned }}=\left(\begin{array}{cccccc}
107.7 & 26.4 & 28.3 & 0 & 0 & 0 \\
26.4 & 107.7 & 28.3 & 0 & 0 & 0 \\
28.3 & 28.3 & 1023.7 & 0 & 0 & 0 \\
0 & 0 & 0 & 111.9 & 0 & 0 \\
0 & 0 & 0 & 0 & 111.9 & 0 \\
0 & 0 & 0 & 0 & 0 & 40.6
\end{array}\right)
$$

\section{Appendix C. Expressions of rotation tensors and orientation distributions functions}

Rotation of a second order tensor, like $\sigma$, around a selected coordinate axis (e.g. $x_{l}$ axis) by an angle of $\vartheta$ is described by the following transformation.

$$
\sigma^{\prime}=R^{T} \cdot \sigma \cdot R, \quad \text { where } R=\left(\begin{array}{ccc}
1 & 0 & 0 \\
0 & \cos \vartheta & \sin \vartheta \\
0 & -\sin \vartheta & \cos \vartheta
\end{array}\right)
$$

Here, $R$ is the rotation matrix and $R^{T}$ is the transpose of it. Using the contracted Voigt notation Eq. (B2), the above transformation can be written in the following form.

$$
\left(\begin{array}{l}
\sigma_{11}{ }^{\prime} \\
\sigma_{22}{ }^{\prime} \\
\sigma_{33}{ }^{\prime} \\
\sigma_{23}{ }^{\prime} \\
\sigma_{13}{ }^{\prime} \\
\sigma_{12}{ }^{\prime}
\end{array}\right)=T_{x 1} \cdot\left(\begin{array}{l}
\sigma_{11} \\
\sigma_{22} \\
\sigma_{33} \\
\sigma_{23} \\
\sigma_{13} \\
\sigma_{12}
\end{array}\right)
$$


The form of $T_{x 1}$ rotation tensor which results in an equivalent transformation with Eq. (C1) is as follows.

$$
T_{x 1}=\left(\begin{array}{cccccc}
1 & 0 & 0 & 0 & 0 & 0 \\
0 & \cos ^{2} \vartheta & \sin ^{2} \vartheta & -2 \cdot \sin \vartheta \cdot \cos \vartheta & 0 & 0 \\
0 & \sin ^{2} \vartheta & \cos ^{2} \vartheta & 2 \cdot \sin \vartheta \cdot \cos \vartheta & 0 & 0 \\
0 & \sin \vartheta \cdot \cos \vartheta & -\sin \vartheta \cdot \cos \vartheta & \cos ^{2} \vartheta-\sin ^{2} \vartheta & 0 & 0 \\
0 & 0 & 0 & 0 & \cos \vartheta & \sin \vartheta \\
0 & 0 & 0 & 0 & -\sin \vartheta & \cos \vartheta
\end{array}\right)
$$

Similar expression is deduced for the rotation of $\sigma$ around the $x_{3}$ axis.

$$
T_{x 3}=\left(\begin{array}{cccccc}
\cos ^{2} \varphi & \sin ^{2} \varphi & 0 & 0 & 0 & -2 \cdot \sin \varphi \cdot \cos \varphi \\
\sin ^{2} \varphi & \cos ^{2} \varphi & 0 & 0 & 0 & 2 \cdot \sin \varphi \cdot \cos \varphi \\
0 & 0 & 1 & 0 & 0 & 0 \\
0 & 0 & 0 & \cos \varphi & \sin \varphi & 0 \\
0 & 0 & 0 & -\sin \varphi & \cos \varphi & 0 \\
\sin \varphi \cdot \cos \varphi & -\sin \varphi \cdot \cos \varphi & 0 & 0 & 0 & \cos ^{2} \varphi-\sin ^{2} \varphi
\end{array}\right)
$$

The equivalent transformation for $\varepsilon$ is similar to Eq. (C2) where corresponding the rotation tensor $\left(T_{x i}^{\varepsilon}\right)$ is expressed by the transpose and the inverse of $T_{x i}$.

$$
\left(\begin{array}{c}
\varepsilon_{11}{ }^{\prime} \\
\varepsilon_{22}{ }^{\prime} \\
\varepsilon_{33}{ }^{\prime} \\
2 \cdot \varepsilon_{23}^{\prime} \\
2 \cdot \varepsilon_{13}^{\prime} \\
2 \cdot \varepsilon_{11}^{\prime}
\end{array}\right)=T_{x i}^{\varepsilon} \cdot\left(\begin{array}{c}
\varepsilon_{11} \\
\varepsilon_{22} \\
\varepsilon_{33} \\
2 \cdot \varepsilon_{23} \\
2 \cdot \varepsilon_{13} \\
2 \cdot \varepsilon_{11}
\end{array}\right), \text { where } T_{x i}^{\varepsilon}=\left(T_{x i}^{T}\right)^{-1}
$$

Taking into account Eqs. (B1), (C2) and (C4), the rotation of the contracted stiffness tensor $\left(C_{i j}\right)$ is calculated as follows which is indicated in Eq. (9).

$$
\sigma^{\prime}=T_{x i} \cdot \sigma=T_{x i} \cdot C \cdot \varepsilon=T_{x i} \cdot C \cdot\left(T_{x i}^{\varepsilon}\right)^{-1} \cdot \varepsilon^{\prime}=\left(T_{x i} \cdot C \cdot T_{x i}^{T}\right) \cdot \varepsilon^{\prime}=C^{\prime} \cdot \varepsilon^{\prime}
$$

The rotation tension $T$, indicated in Eqs. (9), (10), is composed of two rotation tensors around the $x_{1}$ and $x_{3}$ axes in the reference coordinate system shown in Fig. 1.

$$
T=T_{x 3} \cdot T_{x 1}
$$


In Eqs. (9), (10), the $T^{T},\left(T^{-1}\right)^{T}$ mean the transpose of $T$ and $T$ inverse. The inverse of $T$ is calculated according to the below equation:

$$
T^{-1}=T_{x 1}^{-1} \cdot T_{x 3}^{-1}
$$

where $T_{x 1}^{-1}$ and $T_{x 3}^{-1}$ represent the inverse of $T_{x 1}$ and $T_{x 3}$, respectively, generated by a simply substitution of $\varphi$ and $\vartheta$ rotation angles by $-\varphi$ and $-\vartheta$.

The orientation distribution functions of crystalline carbon $\left(\eta_{c}(\vartheta)\right)$ and microvoids $\left(\eta_{v}(\vartheta)\right)$ are defined as follows [21]. These functions depend on only the $\vartheta$ tilt angle due to the rotational symmetry of fibres.

$$
\begin{gathered}
\eta_{c}(\vartheta)=K \cdot \sin ^{\omega} \vartheta \\
K=\frac{\Gamma\left(\frac{\omega+3}{2}\right)}{2 \cdot \pi^{3 / 2} \cdot \Gamma\left(\frac{\omega+2}{2}\right)} \\
\omega=\frac{-\ln 2}{\ln \left(\sin \frac{\pi \cdot(180-H)}{360}\right)} \\
\eta_{v}(\vartheta)=\frac{\omega+1}{4 \cdot \pi} \cdot|\cos \vartheta|^{\omega}
\end{gathered}
$$

where $\Gamma(x)$ is the gamma function having the following property $\Gamma(x+1)=x \cdot \Gamma(x)$ and $H$ is the orientation parameter $(H)$, full-width at half maximum of the (002) azimuthal scan in degrees. 


\section{References}

[1] Krenkel W, Berndt F. C/C-SiC composites for space applications and advanced friction systems. Mater Sci Eng A 2005;421:177-81.

[2] Dalmaz A, Ducret D, El Guerjouma R, Reynaud P, Franciosi P, Rouby D, Fantozzi G, Baboux JC. Elastic moduli of a 2.5D Cf/SiC composite: experimental and theoretical estimates. Comp Sci Technol 2000;60:913-925.

[3] Chen L, Yao X, Cen S. Predictions of elastic property on 2.5D C/SiC composites based on numerical modeling and semi-analytical method. Composites Part B 2015;74:53-65.

[4] Johnson DJ. Structure-property relationships in carbon fibres. J Phys D: Appl Phys 1987;20:286-291.

[5] Minus ML, Kumar S. The processing, properties, and structure of carbon fibers. JOM 2005;57:52-58.

[6] Kriz RD, Stinchcomb WW. Elastic moduli of transversely isotropic graphite fibres and their composites. Exp Mechanics 1979;19:41-49.

[7] Datta S, Ledbetter H, Kyono T. Graphite-fiber elastic constants: determination from ultrasonic measurements on composite materials. In: D.O. Thompson et al. (eds.). Review of progress in quantitative nondestructive evaluation. New York: Springer; 1989. p. 1481-1488.

[8] Dyer SRA, Lord D, Hutchinson IJ, Ward IM, Duckett RA. Elastic anisotropy in unideirectional fibre reinforced composites. J Phys D: Appl Phys 1992;25:66-73.

[9] Piekarczyk W, Kata D. Methodology for determining material constants of anisotropic materials belonging to the transversely isotropic system by ultrasound method. Ultrasonics 2016;71:199-204.

[10] Northolt MG, Veldhuizen LH, Jansen H. Tensile deformation of carbon fibers and the relationship with the modulus for shear between the basal planes. Carbon 1991;29:1267-1279. 
[11] Krucinska I, Stypka T. Direct measurement of the axial Poisson's ratio of single carbon fibres. Comp Sci Technol 1991;41:1-12.

[12] Loidl D, Peterlik H, Müller M, Riekel C, Paris O. Elastic moduli of nanocrystallites in carbon fibers measured by in-situ X-ray microbeam diffraction. Carbon 2003;41:563-570.

[13] Li D, Wang H, Wang X. Effect of microstructure on the modulus of PAN-based carbon fibers during high temperature treatment and hot stretching graphitization. J Mater Sci 2007;42:4642-4649.

[14] Tanaka F, Okabe T, Okuda H, Ise M, Kinloch IA, Mori T, Young RJ. The effect of nanostructure upon the deformation micromechanics of carbon fibres. Carbon 2013;52:372-378.

[15] Miyagawa H, Mase T, Sato C, Drown E, Drzal LT, Ikegami K. Comparison of experimental and theoretical transverse elastic modulus of carbon fibers. Carbon 2006;44:2002-2008.

[16] Kanari M, Tanaka K, Baba S, Eto M. Nanoindentation behavior of a two-dimensional carbon-carbon composite for nuclear applications. Carbon 1997;35:1429-1437.

[17] Diss P, Lamon J, Carpentier L, Loubet JL, Kapsa Ph. Sharp indentation behavior of carbon/carbon composites and varieties of carbon. Carbon 2002;40:2567-2579.

[18] Leatherbarrow A, Wu H. Mechanical behaviour of the constituents inside carbonfibre/carbon-silicon carbide composites characterised by nano-indentation. J Eur Ceram Soc 2012;32:579-588.

[19] Sun Y, Zhao G, Yang F. Anisotropic behavior of the nanoindentation of single carbon fibers. Nanosci Nanotechnol Lett 2014;6:1-5.

[20] Farbos B, Da Costa J-P, Vignoles GL, Leyssale J-M. Nanoscale elasticity of highly anisotropic pyrocarbons. Carbon 2015;94:285-294.

[21] Zhong Y, Bian W, Wang M. The effect of nanostructure on the tensile modulus of carbon fibers. J Mater Sci 2016;51:3564-3573. 
[22] Sauder C, Lamon J. Prediction of elastic properties of carbon fibers and CVI matrices. Carbon 2005;43:2044-2053.

[23] Oliver WC, Pharr GM. Measurement of hardness and elastic modulus by instrumented indentation: Advances in understanding and refinements to methodology. J Mater Res 2004;19:320.

[24] Vlassak JJ, Nix WD. Measuring the elastic properties of anisotropic materials by means of indentation experiments. J Mech Phys Solids 1994;42:1223-1245.

[25] Vlassak JJ, Ciavarella M, Barber JR, Wang X. The indentation modulus of elastically anisotropic materials for indenters of arbitrary shape. J Mech Phys Solids 2003;51:1701-1721.

[26] Delafargue A, Ulm F-J. Explicit approximations of the indentation modulus of elastically orthotropic solids for conical indenters. Int J Solid Struct 2004;41:7351-7360.

[27] Tucker CL, Liang E. Stiffness predictions for unidirectional short-fiber composites: Review and evaluation. Comp Sci Technol 1999;59:655-671.

[28] Li Y, Waas AM, Arruda EM. A closed-form, hierarchical, multi-interphase model for composites - Derivation, verification and application to nanocomposites. J Mech Phys Solids 2011;59:43-63.

[29] Blakslee OL, Proctor DG, Seldin EJ, Spence GB, Weng T. Elastic constants of compressionannealed pyrolytic graphite. J Appl Phys 1970;41:3373-3382.

[30] Qiu YP, Weng GJ. On the application of Mori-Tanaka's theory involving transversely isotropic spheroidal inclusions. Int J Eng Sci 1990;28:1121-1137.

[31] Csanádi T, Németh D, Lofaj F. Mechanical properties of hard W-C coating on steel substrate deduced from nanoindentation and finite element modeling. Exp Mech DOI 10.1007/s11340016-0190-x. 


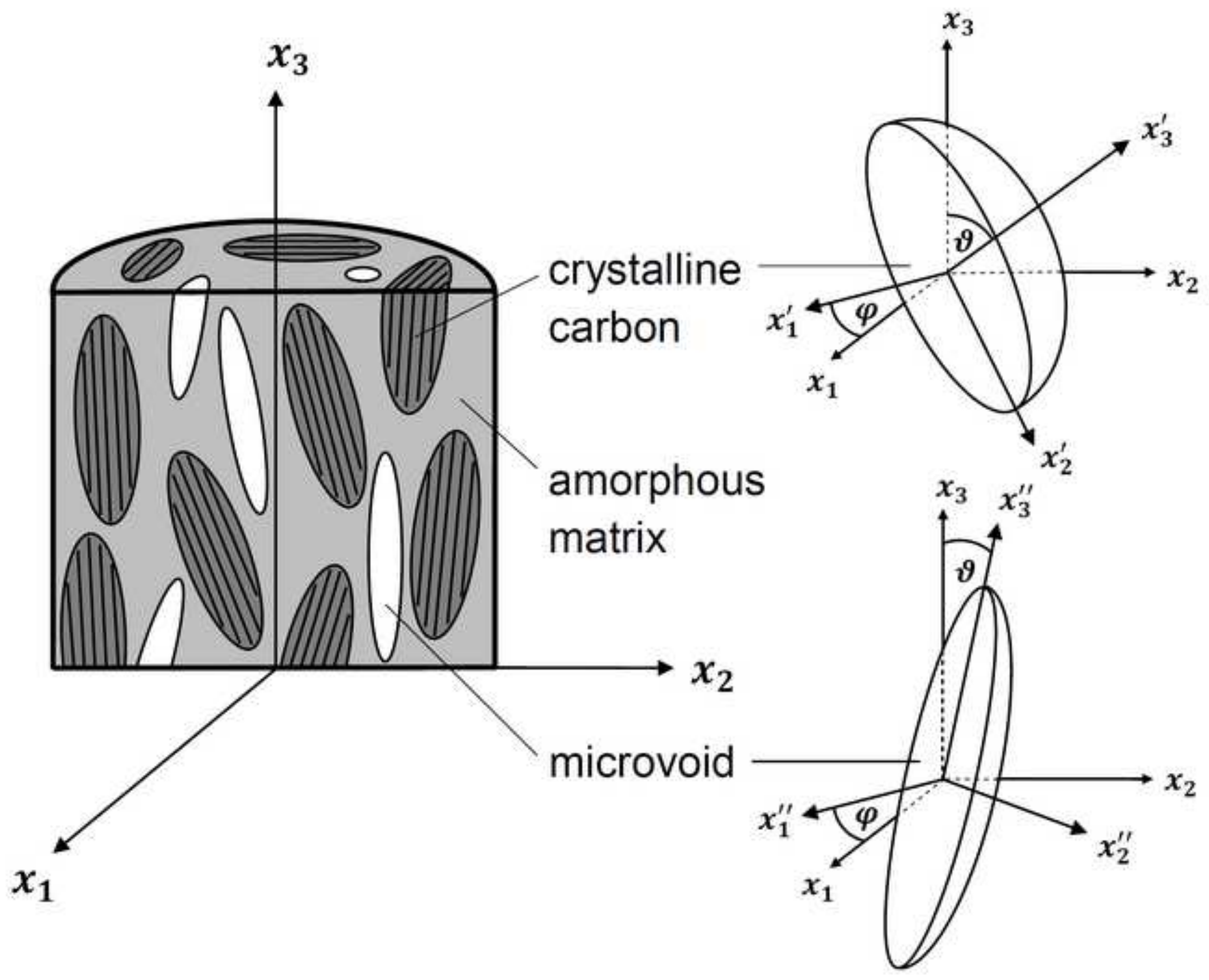



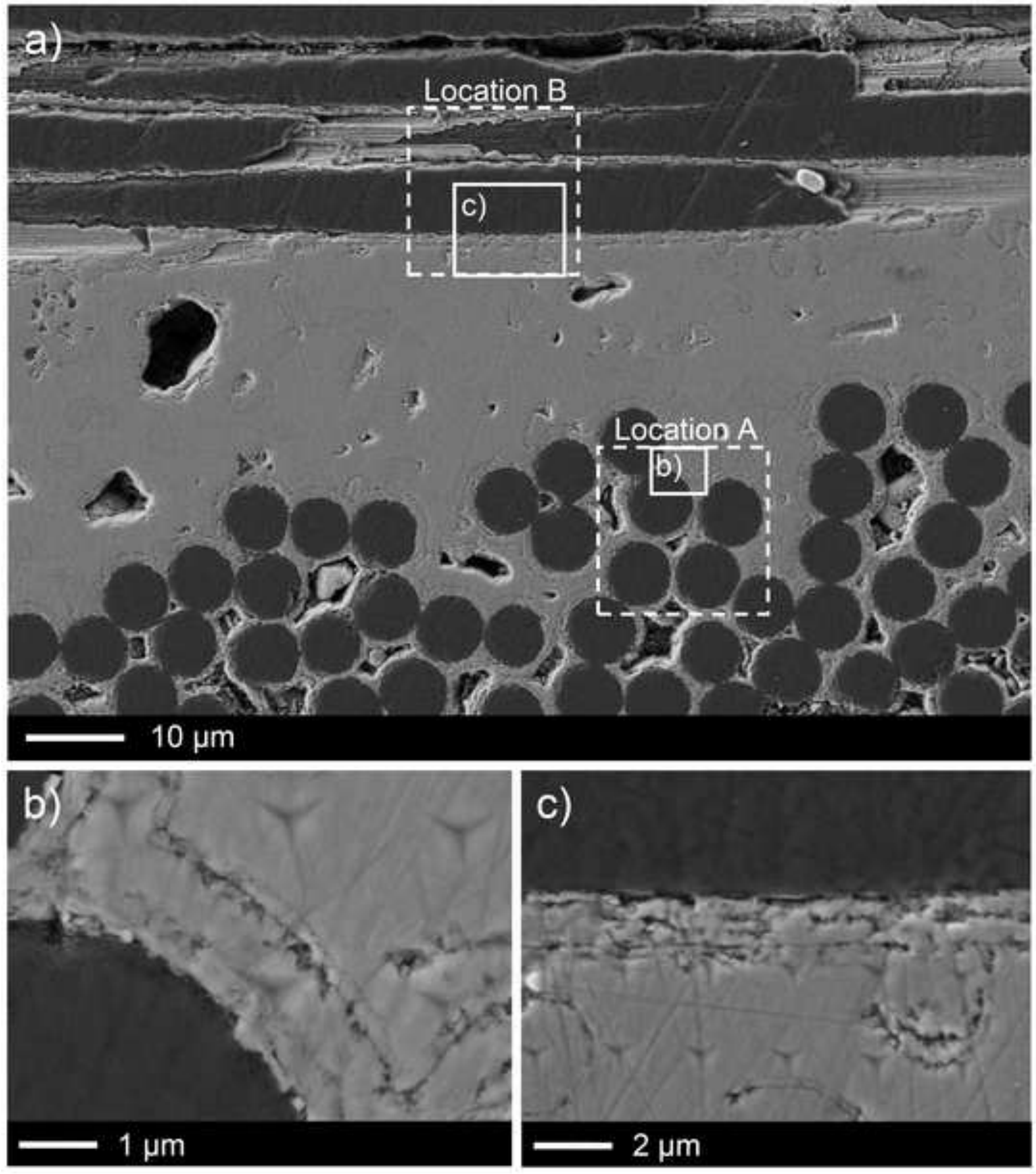

C)
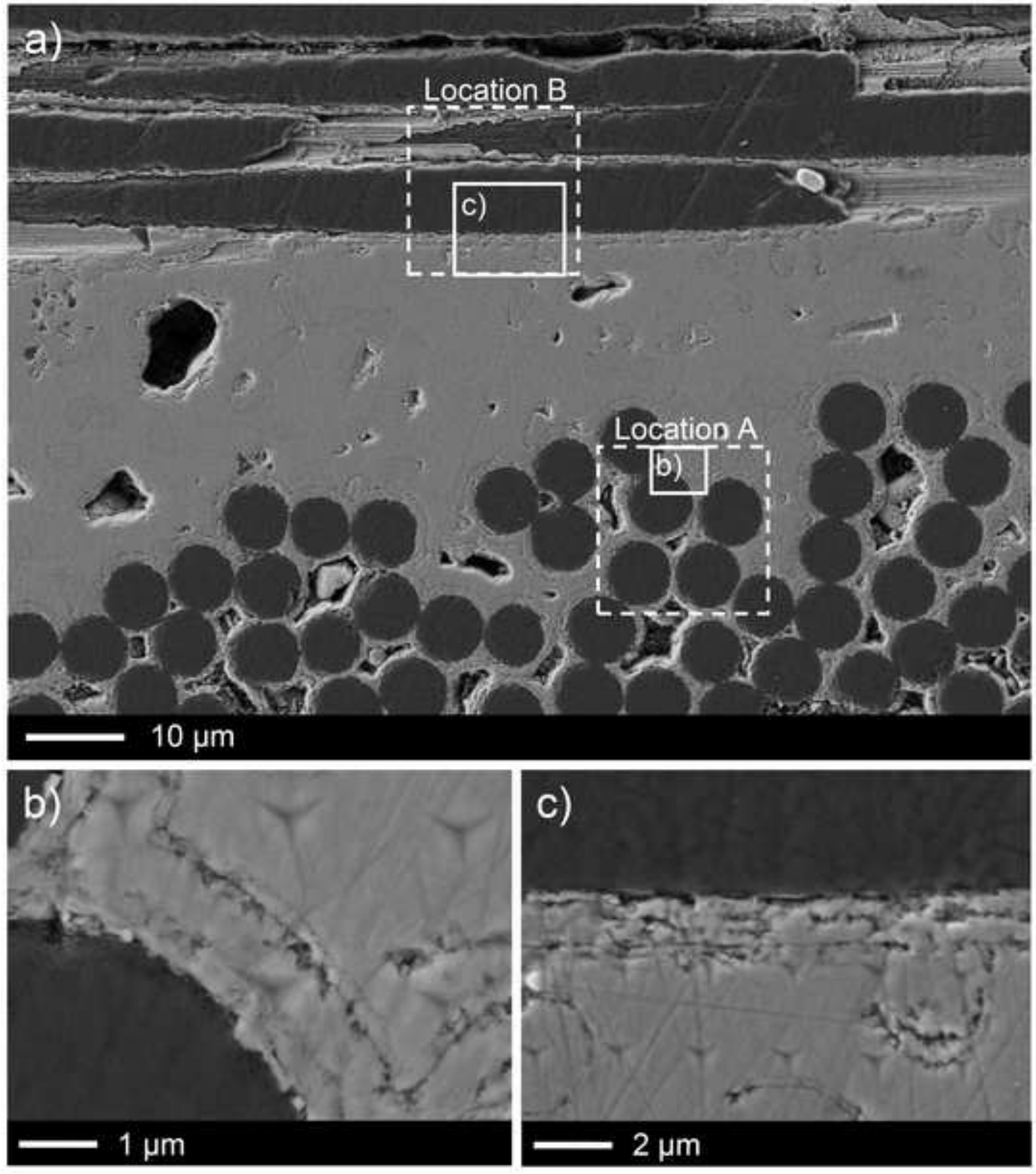
a)

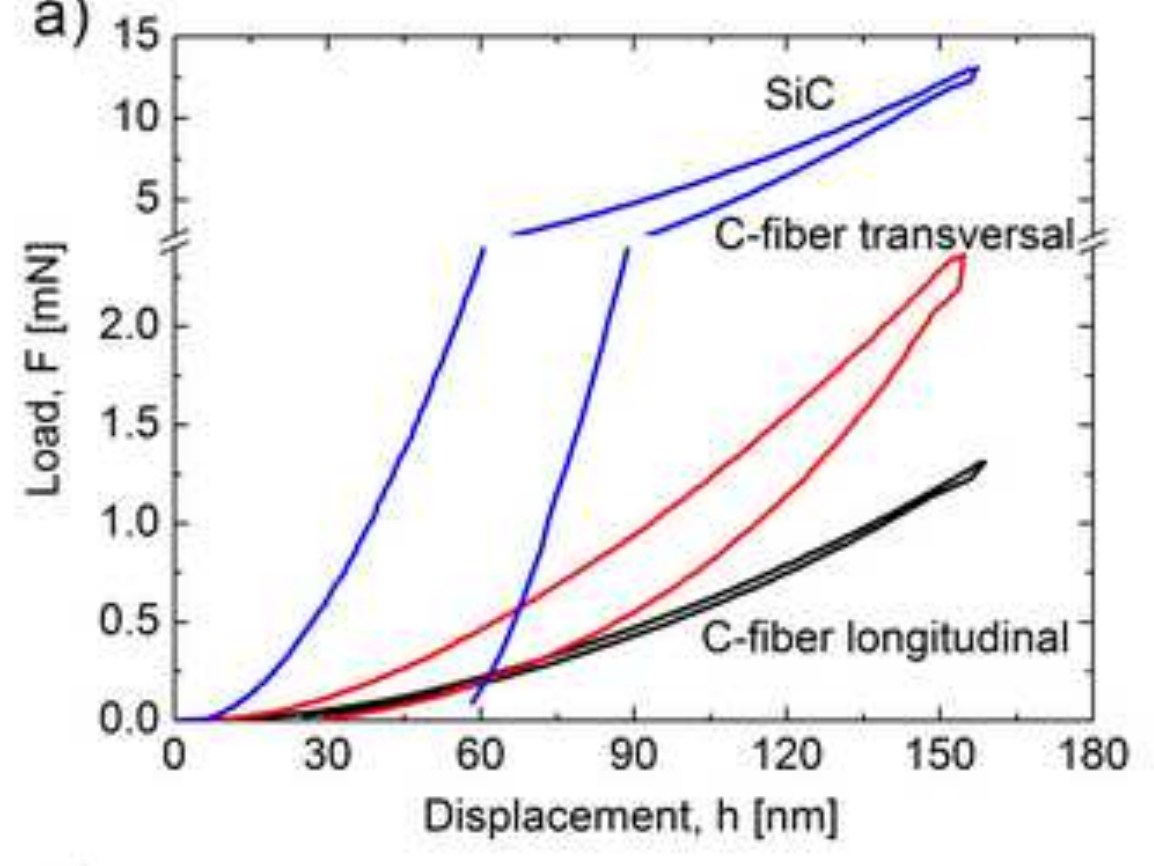

c)

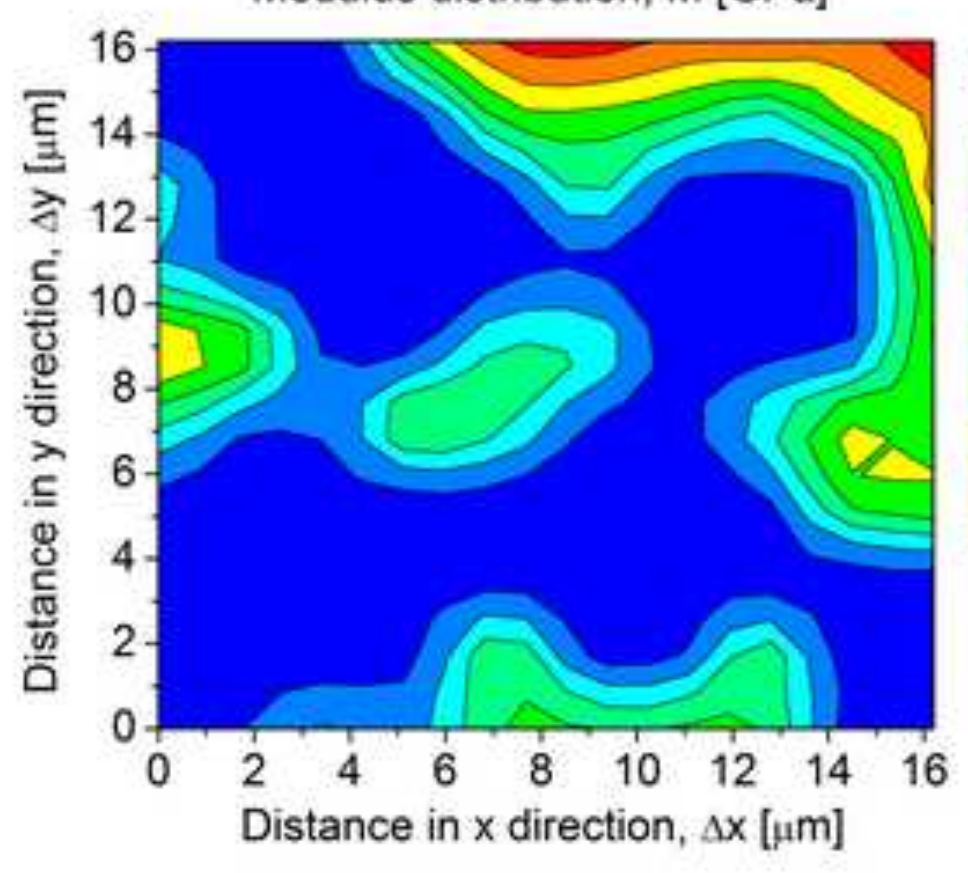

b)

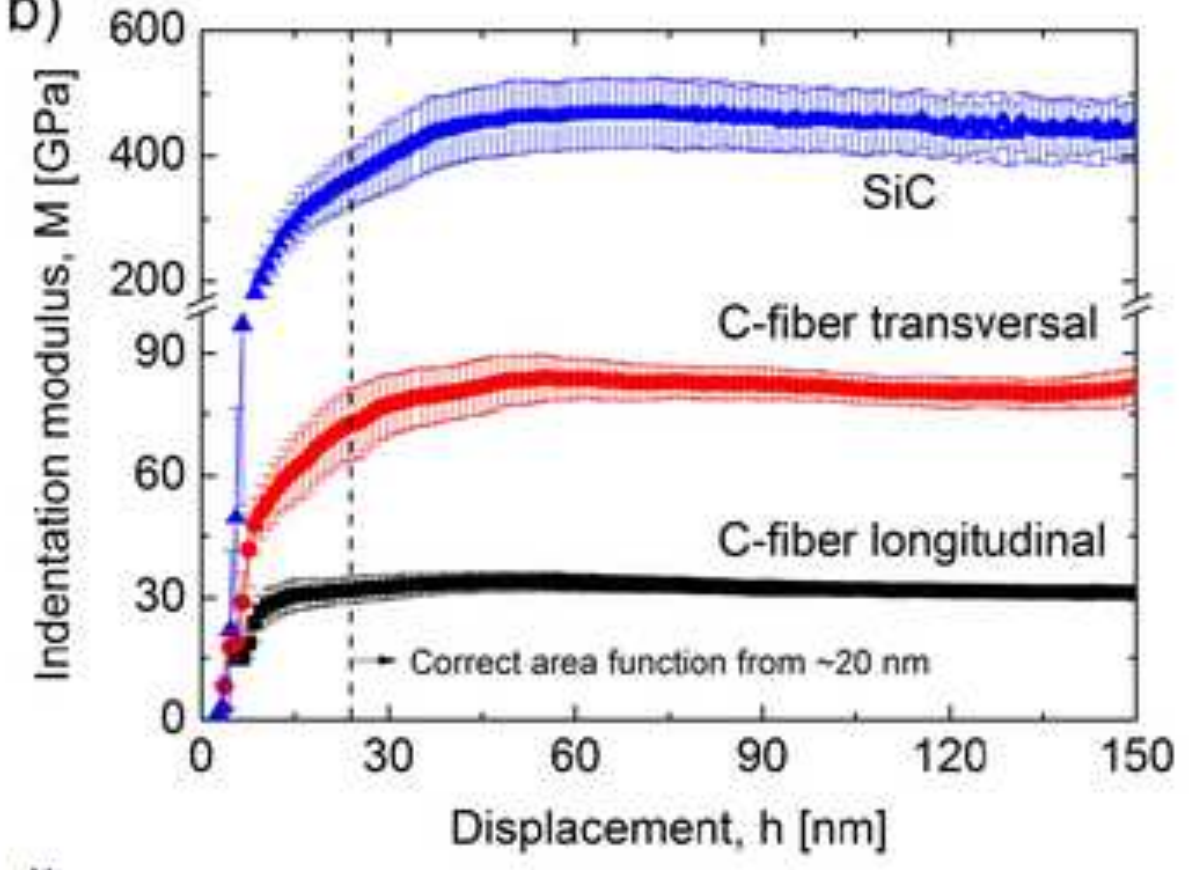

d)

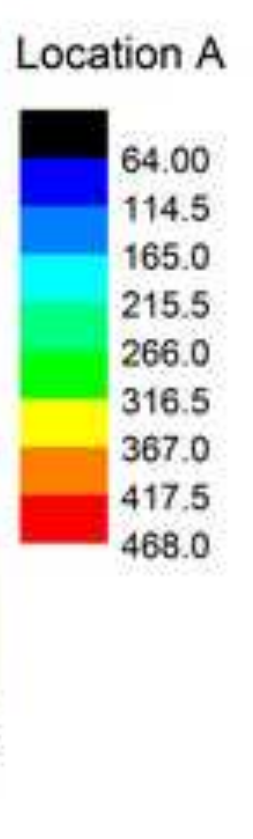

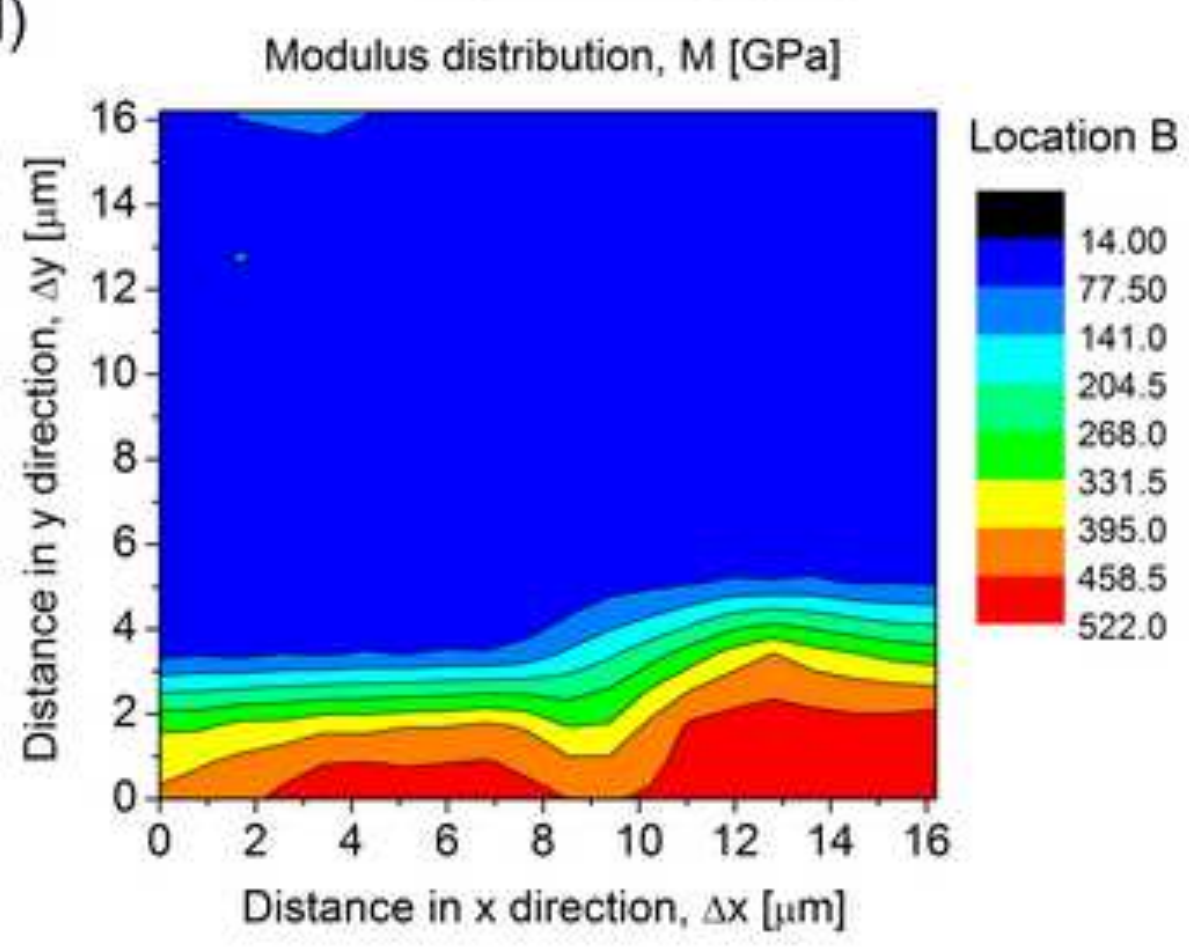




\section{a)}

Carbon fibre

von-Mises

stress [MPa]

5.03e-4 Max

$3,25 \mathrm{e} 4$

3 es

275 e 4

$2.5 \mathrm{e} 4$

2.25 e4

$2 \mathrm{e} 4$

$1,75 \mathrm{e} 4$

$1.5 \mathrm{e} 4$

$1,25 \mathrm{e} 4$

$9.99 \mathrm{e} 3$

$7.5 e 3$

se3

2,5e3

$0,299 \mathrm{Min}$

b) Carbon fibre

SiC matrix

\begin{tabular}{|c|}
\hline $\begin{array}{l}\text { von-Mises } \\
\text { stress [MPa] }\end{array}$ \\
\hline 1,66ed Max \\
\hline $5.8 e 3$ \\
\hline $5,36 e 3$ \\
\hline 4,9103 \\
\hline $4,46 e 3$ \\
\hline $4.02 \mathrm{e} 3$ \\
\hline $3.57 \mathrm{e} 3$ \\
\hline $3.12 \mathrm{e} 3$ \\
\hline $2,68 \mathrm{es}$ \\
\hline $2.23 e 3$ \\
\hline $1,79 e 3$ \\
\hline $1.34 e^{3}$ \\
\hline 893 \\
\hline 446 \\
\hline $0,142 \mathrm{Min}$ \\
\hline
\end{tabular}



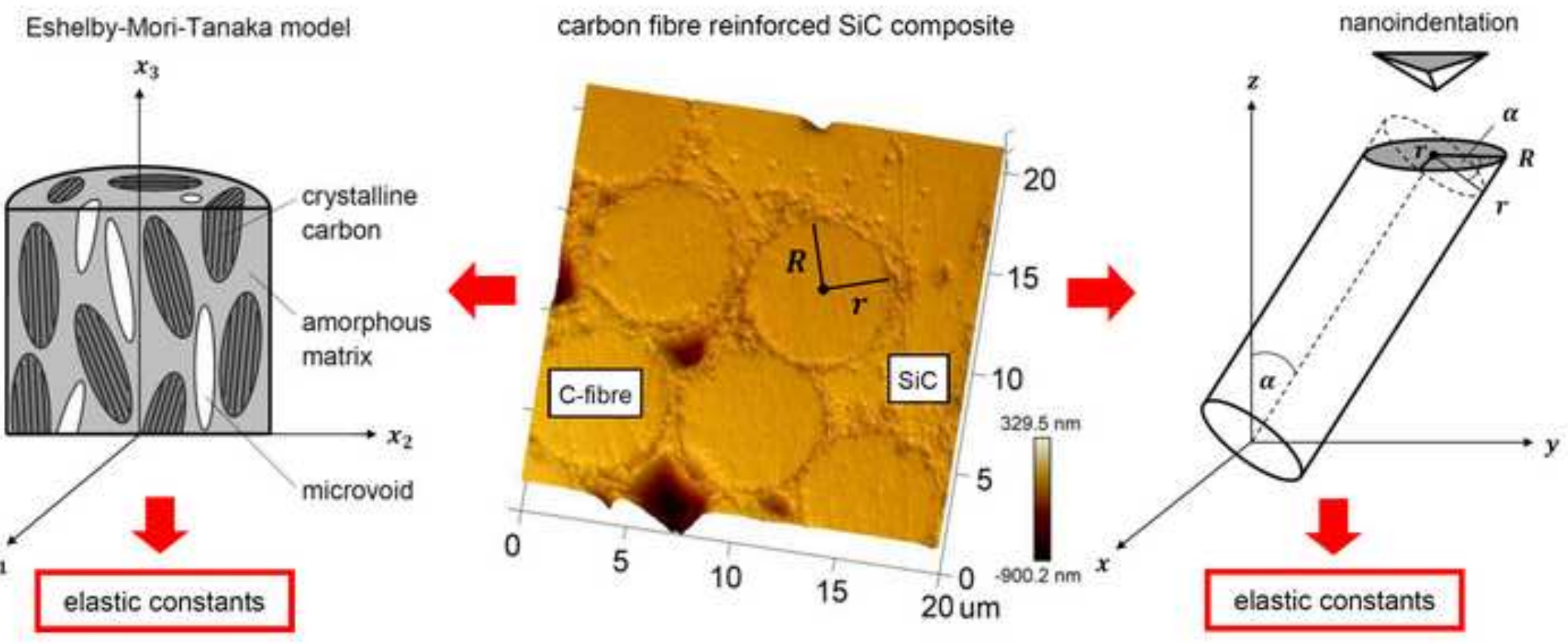


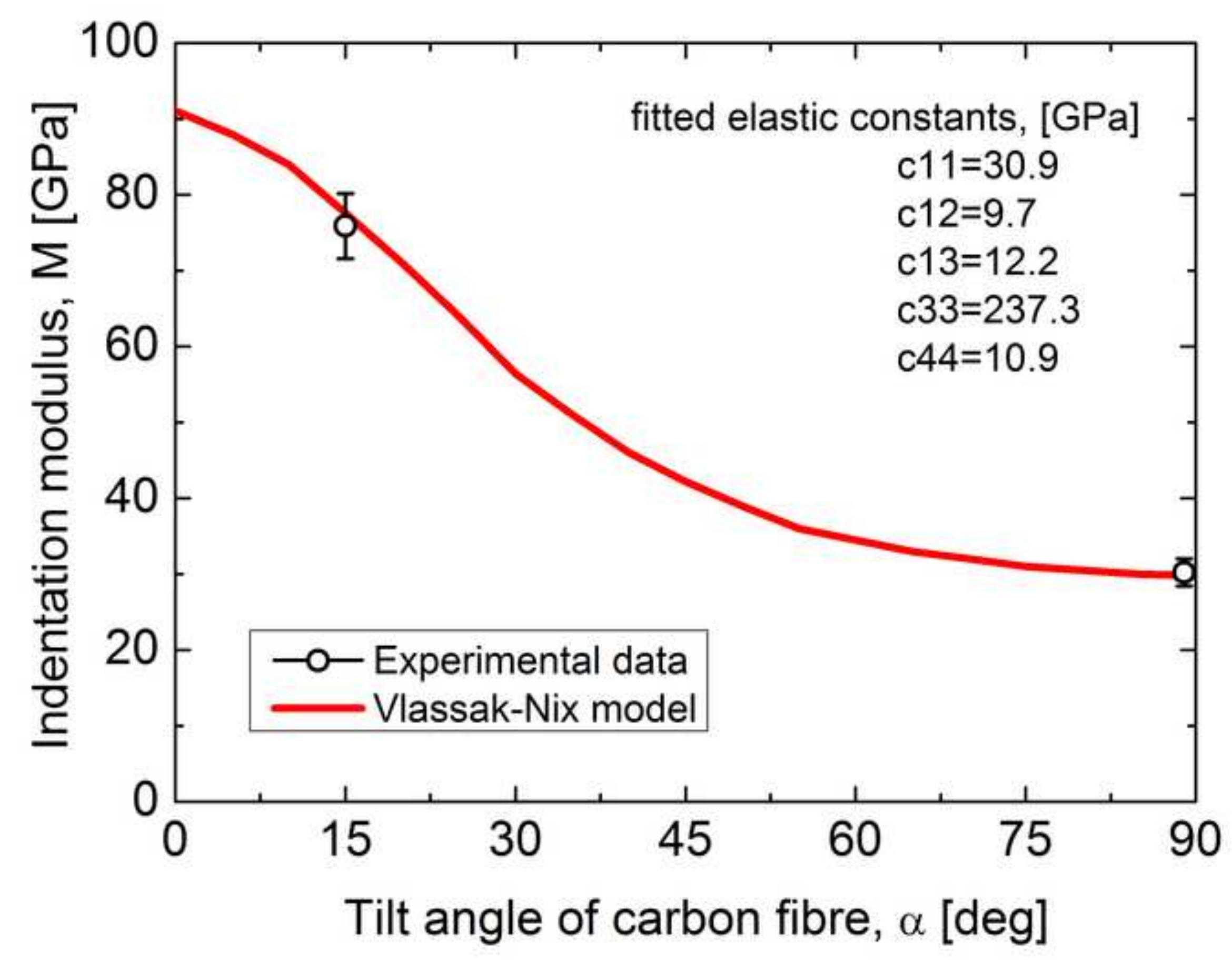




\begin{tabular}{|c|c|c|c|c|c|}
\hline \multirow{2}{*}{ Method } & \multicolumn{5}{|c|}{ Elastic constants, $c_{i j}[\mathrm{GPa}]$} \\
\hline & $c_{11}$ & $c_{12}$ & $c_{13}$ & $c_{33}$ & $c_{44}$ \\
\hline $\begin{array}{l}\text { ultrasound } \\
\text { ref. [7] }\end{array}$ & 20.99 & 7.98 & 9.77 & 225.17 & 17.99 \\
\hline $\begin{array}{l}\text { nanoindentation } \\
\text { this work }\end{array}$ & 30.9 & 9.7 & 12.2 & 237.3 & 10.9 \\
\hline $\begin{array}{l}\text { 2-phase EMT model } \\
\quad\left(\text { reduced } f_{c}\right)\end{array}$ & 151.0 & 50.0 & 54.0 & 260.5 & 74.4 \\
\hline $\begin{array}{l}\text { 2-phase EMT model } \\
\left(\text { reduced } E_{m}\right)\end{array}$ & 91.0 & 30.6 & 28.9 & 244.9 & 62.6 \\
\hline $\begin{array}{l}3 \text {-phase EMT model } \\
\quad\left(\text { reduced } f_{c}\right)\end{array}$ & 116.8 & 33.4 & 37.0 & 249.4 & 71.1 \\
\hline $\begin{array}{l}\text { 3-phase EMT model } \\
\left(\text { reduced } E_{m}\right)\end{array}$ & 79.8 & 23.2 & 21.9 & 240.4 & 63.0 \\
\hline $\begin{array}{l}\text { 2-phase modified } \\
\text { EMT model }\end{array}$ & 43.0 & 13.1 & 25.3 & 253.7 & 31.9 \\
\hline $\begin{array}{l}\text { 3-phase modified } \\
\text { EMT model }\end{array}$ & 41.2 & 10.3 & 18.2 & 243.8 & 35.7 \\
\hline
\end{tabular}

Tab. 1: Elastic constants of T-300 carbon fibre derived from different experimental works and theoretical calculations. 


\begin{tabular}{ccc}
\hline \multirow{2}{*}{ Cross-section of fibre } & \multicolumn{2}{c}{ Indentation modulus, $M[\mathrm{GPa}]$} \\
\cline { 2 - 3 } & conical tip, FEM & Berkovich tip, FEM \\
\hline transversal & 99.01 & 106.34 \\
longitudinal & 25.89 & 27.86 \\
\hline
\end{tabular}

Tab. 2: Simulated indentation modulus values for exact transversal and longitudinal crosssections of carbon fibre by FEM. 


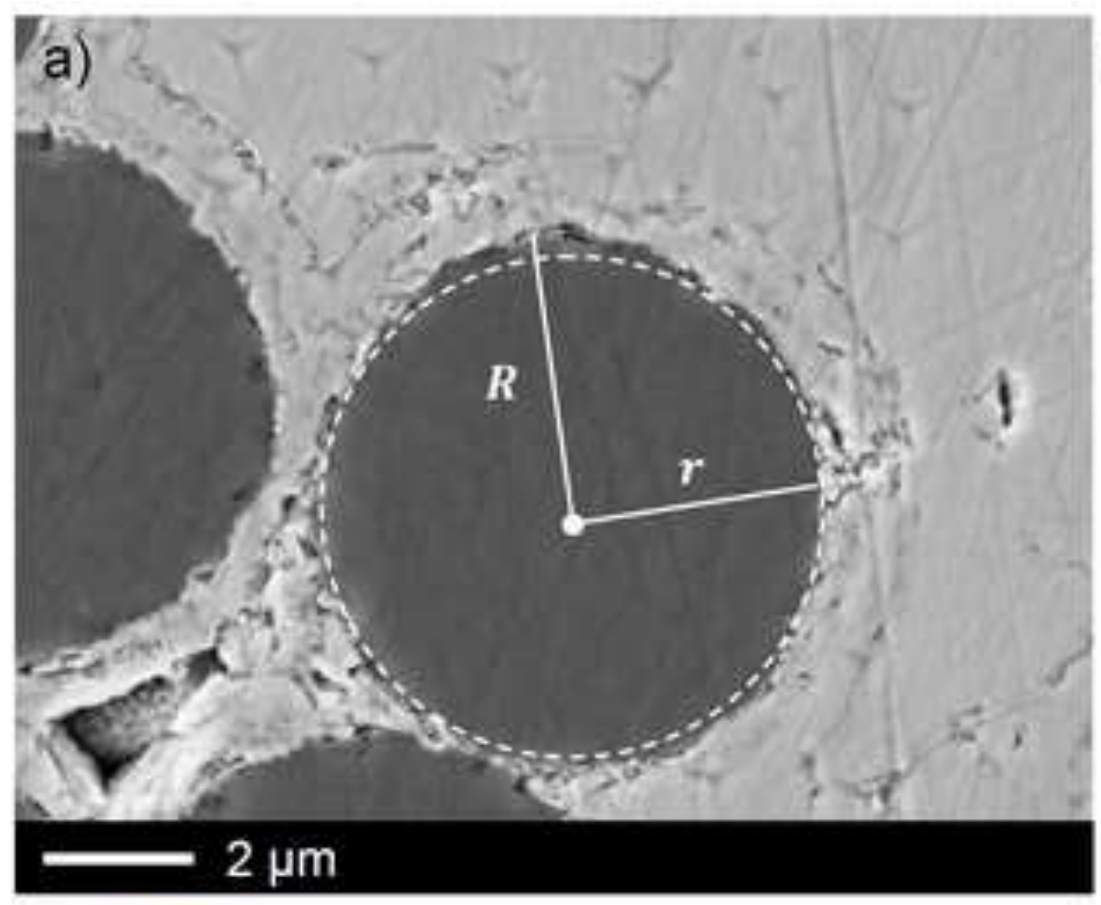

b)

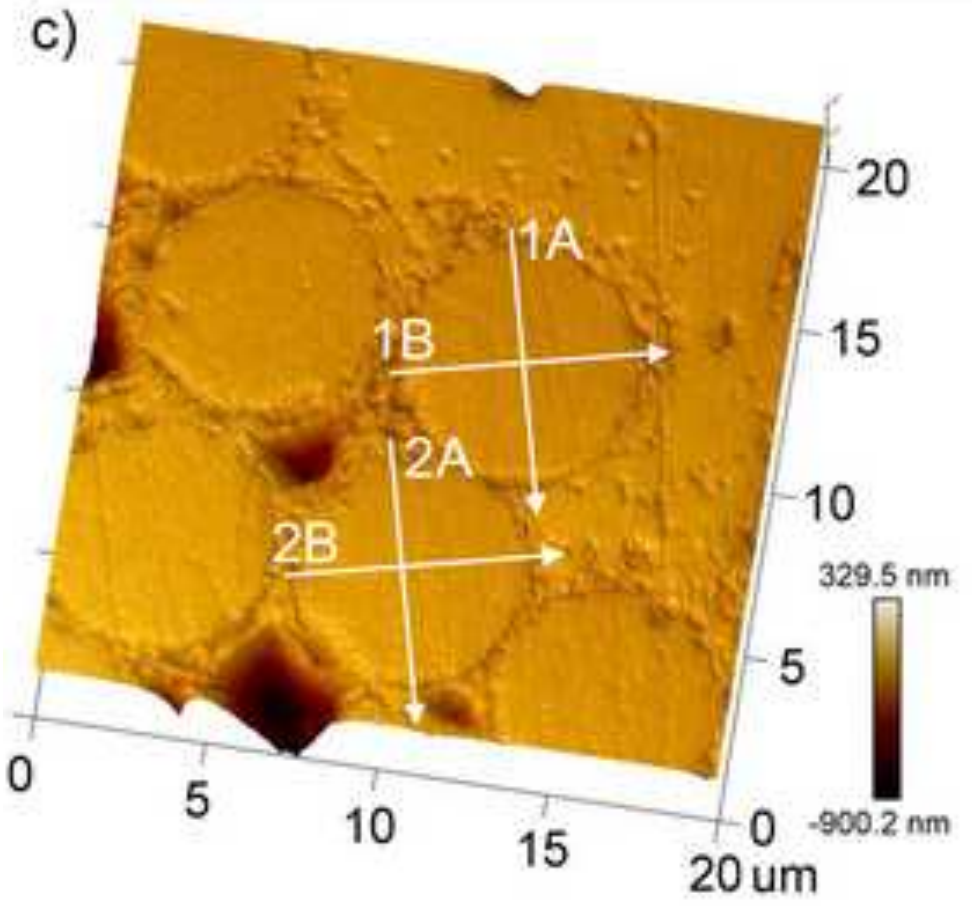

d)

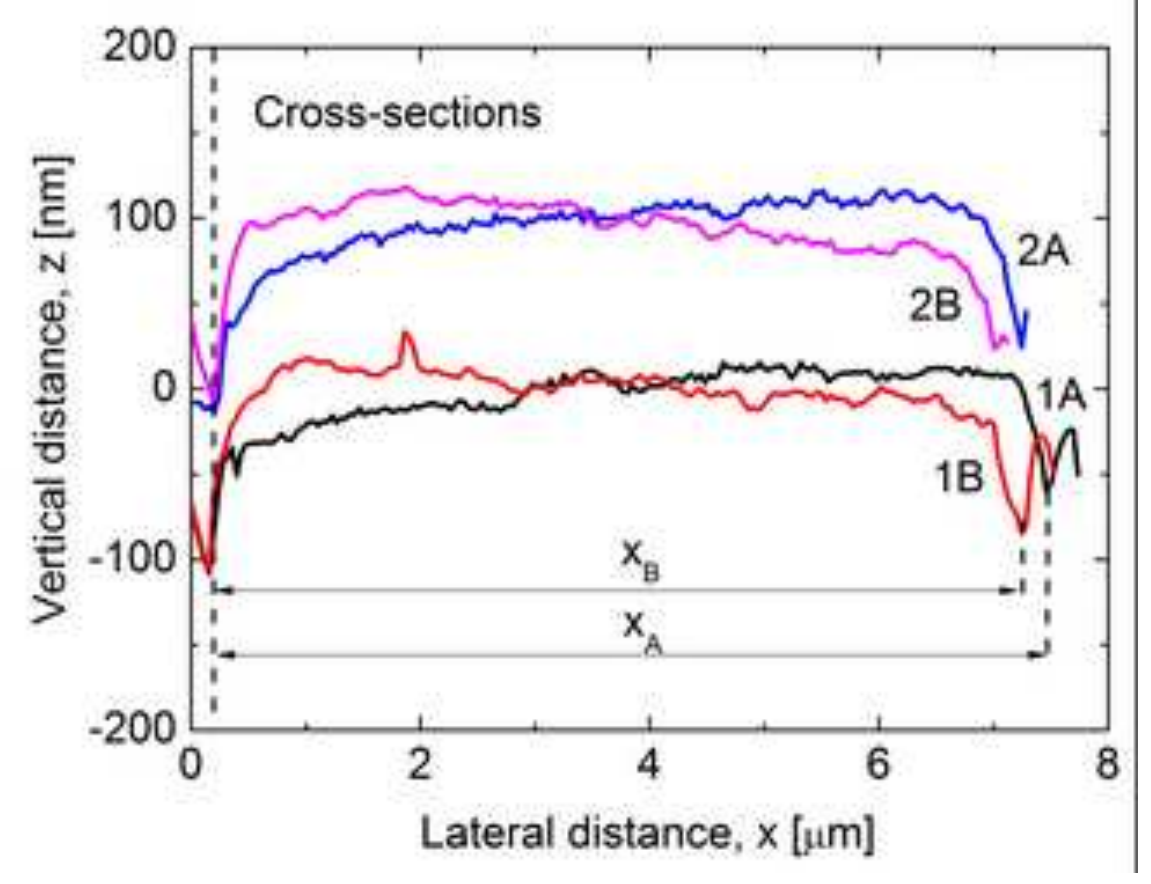

